Florida International University FIU Digital Commons

$5-17-2013$

\title{
Mechanical and Electrical Properties of Single- walled Carbon Nanotubes Synthesized by Chemical Vapor Deposition
}

Yuehai Yang

Florida International University, yyang002@fiu.edu

DOI: $10.25148 /$ etd.FI13080509

Follow this and additional works at: https:// digitalcommons.fiu.edu/etd

Part of the Condensed Matter Physics Commons

\section{Recommended Citation}

Yang, Yuehai, "Mechanical and Electrical Properties of Single-walled Carbon Nanotubes Synthesized by Chemical Vapor Deposition" (2013). FIU Electronic Theses and Dissertations. 946.

https://digitalcommons.fiu.edu/etd/946 


\section{FLORIDA INTERNATIONAL UNIVERSITY}

Miami, Florida

\section{MECHANICAL AND ELECTRICAL PROPERTIES OF SINGLE-WALLED CARBON NANOTUBES SYNTHESIZED BY CHEMICAL VAPOR DEPOSITION}

A dissertation submitted in partial fulfillment of

the requirements for the degree of

DOCTOR OF PHILOSOPHY

in

PHYSICS

by

Yuehai Yang

2013 
To: Dean Kenneth Furton

College of Arts and Sciences

This dissertation, written by Yuehai Yang, and entitled Mechanical and Electrical Properties of Single-walled Carbon Nanotubes Synthesized by Chemical Vapor Deposition, having been approved in respect to style and intellectual content, is referred to you for judgment.

We have read this thesis and recommend that it be approved.

Arvind Agarwal

Yesim Darici

Xuewen Wang

Wenzhi Li, Major Professor

Date of Defense: May 17, 2013

The dissertation of Yuehai Yang is approved.

Dean Kenneth Furton

College of Arts and Sciences

Dean Lakshmi N. Reddi

University Graduate School

Florida International University, 2013 


\section{DEDICATION}

To my beloved wife and daughter

Lian Xue and Hellen Yang. 


\section{ACKNOWLEDGMENTS}

First, I would like to thank my advisor Dr. Wenzhi Li for his tremendous support, encouragement and mentoring throughout my $\mathrm{Ph}$. D study, without which this work would have not been possible. I am truly grateful for his care and guidance at each step of my research and for his tireless help in preparing my publications and presentations. I sincerely appreciate his constructive criticism and tolerance towards me during my study at FIU. His passion and perseverance have had far-reaching influence in me and guided me through difficult times.

I would like to give my special gratitude to Dr. Jin He for his selfless support and help in my electrochemistry research. He has always been available to me to answer my questions and has been not only a great mentor but also an excellent friend. I feel very grateful to Dr. Xuewen Wang for his expert wisdom and help in developing my data analysis skills. I would also like to thank Dr. Arvind Agarwal and Dr. Yesim Darici for their support and advice at various stages of this dissertation writing process.

I am grateful for the help that I received from all of the faculties and staffs in the Department of Physics at FIU. Their expertise and enthusiasm for science and education have inspired me in many different levels.

My graduate studies would not have been the same without academic discussions, values exchanges and emotional support with all my lab mates, classmates and my friends in Miami. I feel fortunate to have their friendship, which have crossed the cultural boundaries, and broadened the horizons in my life.

Finally, I would like to thank my family members in China. Their unconditional loves and support helped me stay focused on my Ph. D study. 


\title{
ABSTRACT OF THE DISSERTATION \\ MECHANICAL AND ELECTRICAL PROPERTIES OF SINGLE-WALLED CARBON NANOTUBES SYNTHESIZED BY CHEMICAL VAPOR DEPOSITION
}

\author{
by
}

Yuehai Yang

Florida International University, 2013

Miami, Florida

\section{Professor Wenzhi Li, Major Professor}

Despite the tremendous application potentials of carbon nanotubes (CNTs) proposed by researchers in the last two decades, efficient experimental techniques and methods are still in need for controllable production of CNTs in large scale, and for conclusive characterizations of their properties in order to apply CNTs in high accuracy engineering. In this dissertation, horizontally well-aligned high quality single-walled carbon nanotubes (SWCNTs) have been successfully synthesized on St-cut quartz substrate by chemical vapor deposition (CVD). Effective radial moduli ( $\left.\mathrm{E}_{\text {radial }}\right)$ of these straight SWCNTs have been measured by using well-calibrated tapping mode and contact mode atomic force microscopy (AFM). It was found that the measured $\mathrm{E}_{\text {radial }}$ decreased from 57 to $9 \mathrm{GPa}$ as the diameter of the SWCNTs increased from 0.92 to $1.91 \mathrm{~nm}$. The experimental results were consistent with the recently reported theoretical simulation data. The method used in this mechanical property test can be easily applied to measure the mechanical properties of other low-dimension nanostructures, such as nanowires and nanodots. The characterized sample is also an ideal platform for electrochemical tests. The electrochemical activities of redox probes $\mathrm{Fe}(\mathrm{CN})_{6}{ }^{3-/ 4-}, \mathrm{Ru}\left(\mathrm{NH}_{3}\right)_{6}{ }^{3+}, \mathrm{Ru}(\mathrm{bpy})_{3}{ }^{2+}$ and protein cytochrome $\mathrm{c}$ have been 
studied on these pristine thin films by using aligned SWCNTs as working electrodes. A simple and high performance electrochemical sensor was fabricated. Flow sensing capability of the device has been tested for detecting neurotransmitter dopamine at physiological conditions with the presence of Bovine serum albumin. Good sensitivity, fast response, high stability and anti-fouling capability were observed. Therefore, the fabricated sensor showed great potential for sensing applications in complicated solution. 


\section{TABLE OF CONTENTS}

CHAPTER

PAGE

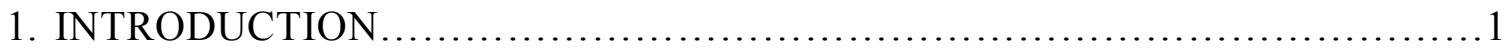

1.1 A brief history of carbon nanotube research..................................

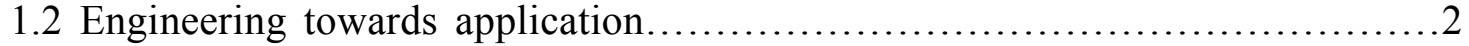

1.3 SWCNT structure and specification.........................................

1.4 Investigations of mechanical and electrical properties on individual CNTs......5

1.5 Investigation of CNTs in electrochemistry ................................. 8

1.6 Remaining issues of CNT research ..........................................

2. REVIEW OF SYNTHESIS METHODS OF CARBON

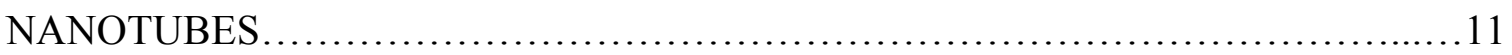

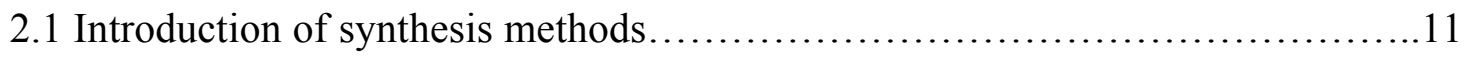

2.2 Synthesis of single-walled carbon nanotube.................................. 16

2.3 Alignment control of SWCNTs on the substrate surface.................... 20

3. SYNTHESIS AND CHARACTERIZATION OF SINGLE-WALLED

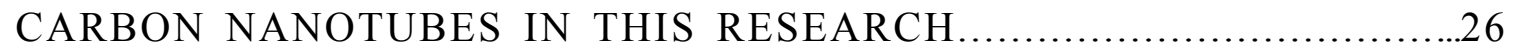

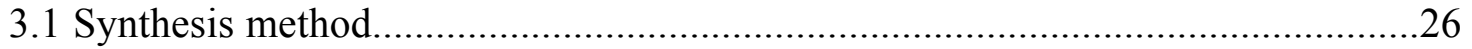

3.2 Characterization of single-walled carbon nanotubes........................................27

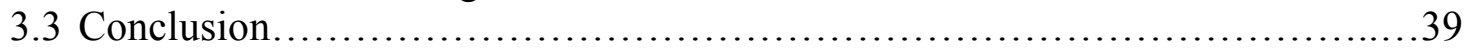

4. RADIAL ELASTICITY MEASUREMENTS OF SINGLE-WALLED CARBON NANOTUBES BY ATOMIC FORCE MICROSCOPY ..............................41



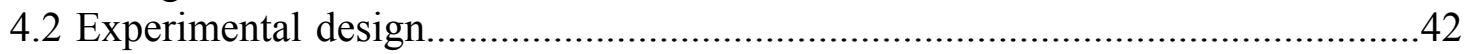

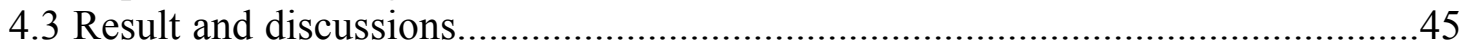



5. ELECTRICAL/ELECTROCHEMICAL PROPERTIES AND THE BIOSENSING OF SINGLE-WALLED CARBON NANOTUBES.....................54

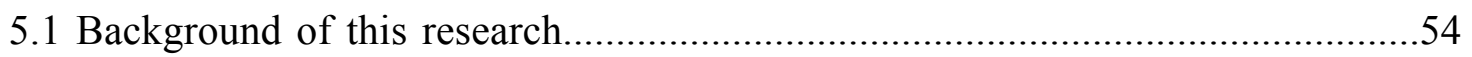

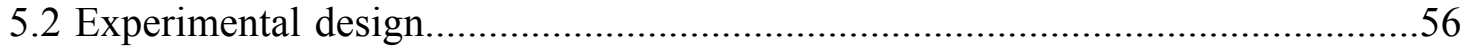

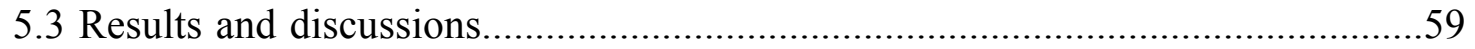



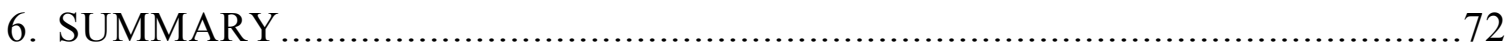

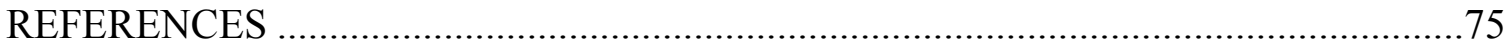

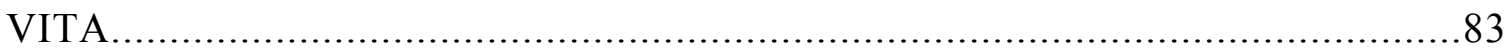




\section{LIST OF FIGURES}

FIGURE

PAGE

1: Unrolled hexagonal lattice of a $(3,1)$ single-walled carbon nanotube as a graphene sheet.

2: A top view SEM image of synthesized high quality, high density vertically aligned CNTs by our CVD system. Inset: TEM image of individual CNTs on same sample shows inner diameters of $\sim 4.5 \mathrm{~nm}$. 16

3: The experimental components of the arc-discharge technique for SWCNT production. 17

4: The schematics of the experimental set-up for laser ablation. 18

5: The schematics of experimental set-up of our chemical vapor deposition system.

6: Flow orientated millimeter-long SWCNTs. (a) A pristine sample. (b) A sample patterned with gold electrodes.

7: (a) A typical SEM image of as-grown SWCNTs. (b) A topographic AFM image of my sample.

8: (a) Average diameter measurement over the length direction of the individual SWCNTs in the AFM image. (b) The average height profiles of the SWCNTs in the image. (c) The diameter distribution of 80 SWCNTs

9: (a) SWCNTs synthesized on original St-cut quartz without pre-growth annealing. (b) SWCNTs synthesized on St-cut quartz annealed for 4 hours (c) 8 hours, and (d) 20 hours.

10: (a) An AFM image of step features along the [100] direction on St-cut quartz surface after 20 hours thermal annealing. (b) A 3-D AFM image of the same step features.

11: From image (a) to (f), a continuous imaging process of $48 \mu \mathrm{m}$ along the growth direction of sample 1 .

12: From image (a) to (f) in Fig. 11, the number of nanotubes declines exponentially from 162 to 2 along the growth direction of sample 1

13: From image (a) to (d), a continuous imaging process of $24 \mu \mathrm{m}$ along the growth direction of sample 2 .

14: (a) Individual SWCNT turned 180 degrees for multiple times on the substrate. (b) SWCNT segments, grown in the random direction, functioned as 
nano-barriers that stopped the growth SWCNT grown along the [100] direction. (c) An AFM image of a nano-barrier.

15: Raman spectra of two pristine samples give distinctive peaks at G-band but no obvious peaks at D-band.

16: The schematic of the cross-section of AFM tip-SWCNT-substrate system when the AFM tip (with $R_{\text {tip }} \approx 20 R_{\text {cnt }}$ ) is going across the ridge of a SWCNT. It is assumed that the thickness of the native oxide layer on the AFM is $\sim 2 R_{\text {cnt }}$.

17: (a): High resolution tapping mode AFM image of the SWCNT. (b) and (c): contact mode images of the same region shown in panel (a), the white boxes show where the radial modulus measurement taking place for individual CNT. (d), (e) and (f): The averaged cross-sections of the boxed volumes in panel (a), (b) and (c), respectively. Height color bars are $0-4 \mathrm{~nm}$ in all three images. The contact forces are $0,7.4$, and $13.1 \mathrm{nN}$ for scanning image (a) (free-amplitude tapping), (b), and (c), respectively.

18: The force calibration curve of our AFM system 47

19: The measured $\Delta \mathrm{h}$ vs. $\mathrm{F}_{\mathrm{z}}$ as well as the fitting to the experimental data by using the power function relation $\Delta h \propto \mathrm{F}_{\mathrm{z}}{ }^{2 / 3}$. From the top fitted curve to the bottom one, the diameter of SWCNTs decreases from 1.91 to $0.42 \mathrm{~nm}$.

20: The data of measured $\mathrm{E}_{\text {radial }}$ vs. $\mathrm{R}_{\mathrm{cnt}}$ (black squares) compares with nanoindentation experimental data (blue dots, see [8]) and modified MSM calculation data (red triangles, data courtesy of Mr. Y.L. Liu).

21: The renormalized data in the plot of $\mathrm{Fd}^{3 / 2}\left(2 \mathrm{R}_{\text {tip }}\right)^{-1 / 2}$ vs. Radial strain.........................50

22: $\Delta \mathrm{h}$ and $\Delta \eta$ as different functions of $\mathrm{Fz}$ for a SWCNT with $0.92 \mathrm{~nm}$ diameter. ............51

23: The data of $(\Delta \mathrm{h}-\Delta \eta) / \Delta \eta$ vs. diameter for 7 different SWCNTs.................................... 52

24: (a) Scheme of the device and electrochemical measurement connections. (b) The top view of the fabricated device. 58

25: (a) A typical IV characteristics of a 2-terminal sample. (b) The scheme of the fabricated MOS-FEM based on the synthesized sample. (c) The applied $\mathrm{V}_{\text {sd }}$ (blue) and $\mathrm{V}_{\mathrm{g}}$ (red). (d) 5 source-drain IV curves, indicated by 5 colors.

26: (a) A typical cyclic voltammogram (CV) of $25 \mu \mathrm{M}$ Potassium Ferrocyanide in $1 \mathrm{M} \mathrm{KCl}$. (b) $\mathrm{CV}$ of $25 \mu \mathrm{M} \mathrm{Ru}\left(\mathrm{NH}_{3}\right)_{6} \mathrm{Cl}_{3}$ in $1 \mathrm{M} \mathrm{KCl}$. (c) $\mathrm{CV}$ of $25 \mu \mathrm{M}$ Potassium Ferricyanide in $1 \mathrm{M} \mathrm{KCl}$. For (a), (b) and (c), the red curve is the fitting curve of oxidation current using equation (1). The black curve is a control results in pure $1 \mathrm{M} \mathrm{KCl}$. The potential sweep rate for both CVs is $200 \mathrm{mV} / \mathrm{s}$. (d) $\mathrm{CV}$ of $20 \mu \mathrm{M}$ cyc in $1 \mathrm{M} \mathrm{KCl}$ measured before (blue) and after (red) rinsing the CNT thin film surface. The potential scan rate is $25 \mathrm{mV} / \mathrm{s}$. 
27: (a) Cyclic voltammograms of $25 \mu \mathrm{M} \mathrm{Ru}$ (bpy) ${ }_{3} \mathrm{Cl}_{2}$ in $1 \mathrm{M} \mathrm{KCl}$ measured at different potential scan rates. (b) The plot of the cathodic peak current $\left(\mathrm{i}_{\mathrm{pc}}\right)$ and anodic peak current $\left(\mathrm{i}_{\mathrm{pa}}\right)$ versus the square root of the potential scan rate $\left(\mathrm{v}^{1 / 2}\right)$. The solid lines are linear fits to the experimental data.

28: (a) A typical cyclic voltammetry (CV) of $25 \mu \mathrm{M}$ dopamine in $1 \mathrm{M} \mathrm{KCl}$. The red curve is the fitting curve using equation (1). The black curve is a control results in pure $1 \mathrm{M} \mathrm{KCl}$. The sweep rate is $200 \mathrm{mV} / \mathrm{s}$. (b) Electrochemical (EC) current at $\mathrm{E}=0.6 \mathrm{~V}$ as a function of dopamine concentration in $1 \mathrm{x}$ PBS buffer. The inset shows the linear relationship between EC current and dopamine concentration at low concentration range. (c) The EC current vs. dopamine concentration with (red sphere) and without (blue sphere) 3\% (w/w) BSA in $1 \times$ PBS buffer. The inset shows typical CVs of $1 \mathrm{mM}$ dopamine with (red line) and without (blue line) $3 \%(\mathrm{w} / \mathrm{w}) \mathrm{BSA}$ in $1 \mathrm{xPBS}$ buffer. (d) The EC current (at $\mathrm{E}=0.6$ ) versus time when various concentration of dopamine is added into the solution $(3 \%(\mathrm{w} / \mathrm{w})$ BSA in $1 \mathrm{xPBS}$ buffer). The flow rate is a $10 \mathrm{~mL}$ per hour.

29: (a) A side-view scheme of the flow sensing cell. (b) The EC current ( $\mathrm{E}=0.6$ ) versus time when 2,5 and $8 \mu \mathrm{M}$ of dopamine is added into the solution (3\% $(\mathrm{w} / \mathrm{w}) \mathrm{BSA}$ in $1 \mathrm{xPBS}$ buffer). The flow rate is at $0.5 \mathrm{~mL}$ per hour 


\section{CHAPTER 1}

\section{Introduction}

\subsection{A brief history of carbon nanotube research}

Carbon nanotube (CNT) has a seamless cylindrical shape. As in graphite, carbon atoms are arranged in a hexagonal way that replicate throughout the structure of a CNT. With a diameter of a few nanometers, a CNT can be centimeters long [1], giving it a higher aspect ratio compared to any other material. The fundamental cylindrical structure forms the building block of multiwalled carbon nanotubes (MWCNT), which can be viewed as multiple single-walled carbon nanotubes (SWCNTs) with different diameters coaxially situate within each other. The inter-tube spacing between the concentric walls

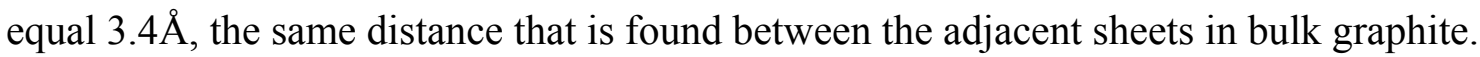

The MWCNT is commonly believed to be first discovered in 1991 by Sumio Iijima in Japan [2] using an arc-discharge evaporation method. Ijiima et al. continued their work and discovered SWCNTs in 1993 [3] simultaneously with Bethune et al. at the IBM research division in California USA [4]. The discovery of these unique structures led to a highly motivated effort of studying their properties in the past two decades, where the conducted research led to very optimistic and promising results. It is suggested that CNTs can provide high stiffness [5], high electrical conductivity, high thermal conductivity $[6,7]$ and high current density, while having a low density. In addition, CNTs also are light in weight, chemically inert, and mechanically flexible [8]. The extensive study of the properties of CNT revealed extraordinary axial strength of this "ultimate fiber". The axial strength of CNT can be attributed to the C-C covalent bond, the strongest bond in nature, which essentially forms the CNT structure. It has been 
shown that the Young's modulus of CNT in the axial direction is in the range of 270-950 $\mathrm{GPa}$ and its tensile strength is of 11-63 GPa, which makes CNT one of the strongest materials on earth. Also, CNTs are efficient conductors of heat [9]. At room temperature, CNTs have the thermal conductivity as high as $6600 \mathrm{~W} / \mathrm{mK}[10]$.

\subsection{Engineering towards application}

With such special properties, carbon nanotubes have varieties of application possibilities in the fields of material science, electronics, biology, medicine, electrochemistry, and field emission [11]. The specific potential application includes but is not limited to reinforcements materials in alloys, nanotransistors in circuits, field emission displays, and artificial muscles [12]. In order to realize these possible applications, the majority of ongoing research has emphasized understanding, controlling and manipulating physical properties of CNTs accurately. The current public interest on CNTs rests on adding them into other materials to change the mechanical and electrical properties of the latter. The newly formed composite will take on some of the properties of the CNTs. For example, CNTs can be mixed into the matrix polymer to increase the electrical conductivity, thermal conductivity, bulk strength, as well as strength to weight ratio of the newly formed polymer composite. The realized applications of CNT composite include sporting equipment, electronic displays, auto parts, thermal grease, yarns, etcetera.

\subsection{SWCNT structure and specification}

One of the most intriguing facts about CNT which has been discovered is its unique electrical property. SWCNTs can have metallic or semiconducting property. Therefore, SWCNTs are ideal candidates for novel molecular devices because their 
electronic properties depend on their geometry rather than on the doping of impurities, resulting in high thermal stabilities. Hence, it is critical to specify the SWCNT geometry in an effective way.

A SWCNT can be viewed as a rolled up graphene sheet where atoms are arranged in a hexagon. It is characterized by a chiral vector $\mathrm{C}$ which is defined in Eqn.1.1 by two integers $(n, m)$ and graphite vectors $a$ and $b$. The chiral vector $C$ is described in Fig. 1.

$\mathbf{C}=\mathrm{na}+\mathrm{mb}=(\mathrm{n}, \mathrm{m})$ where $n$ and $m$ are integers and $0 \leqq m \leqq n$.

As shown in Figure 1, the structure of the SWCNT is exclusively determined by the chiral vector $\mathrm{C}$, which connects two random carbon atoms in the graphene sheet. The integer pair (n, m) can be utilized to specify a SWCNT. The carbon nanotube has a diameter specified by Eqn. 1.2.

$\mathrm{D}=|\mathbf{C}| / \pi=\alpha\left(\mathrm{n}^{2}+\mathrm{nm}+\mathrm{m}^{2}\right)^{1 / 2} / \pi$

where $\alpha=|\mathbf{a}|=|\mathbf{b}|=2.49 \AA$, and refers to the lattice constant of graphite.

The chiral angle $\varnothing=\operatorname{arcos} \frac{2 m+n}{2 \sqrt{n^{2}+n m+m^{2}}}$

Perpendicular to the chiral vector $\mathrm{C}$, a translation vector $\mathrm{T}$ shares one ending atom with the vector $\mathrm{C}$ and connects with the closest atom which is equivalent to this ending atom of vector $\mathrm{C}$. The translation vector $\mathrm{T}$ is defined as the unit vector of this 1 dimensional nanotube. The nanotube is visually formed by rolling this rectangular sheet across $\mathrm{T}$ so that the two end of vector $\mathrm{C}$ coincide each other.

The SWCNTs can be categorized into 3 groups [13]: When $\mathrm{n}=0$, the nanotube $(0$, m) is called a zigzag SWCNT, when $n=m$, the nanotube $(n, n)$ is called an armchair SWCNT; and in all other scenarios, the nanotube is called a chiral nanotube. 
Giving this specification, if the n-m is a multiple of 3 for a given SWCNT (n, m), the nanotube is semi-metallic with a small band gap. However if $n=m$, then it shows metallic behavior, otherwise, the nanotube is moderately semiconducting. According to the definition of arm chair SWCNT, all arm chair SWCNTs are metallic.

By using the index (n, m), we can specify the structure of the SWCNT and determine important parameters of the SWCNT such as the diameter and chirality which can further determine the electrical properties of the SWCNT.

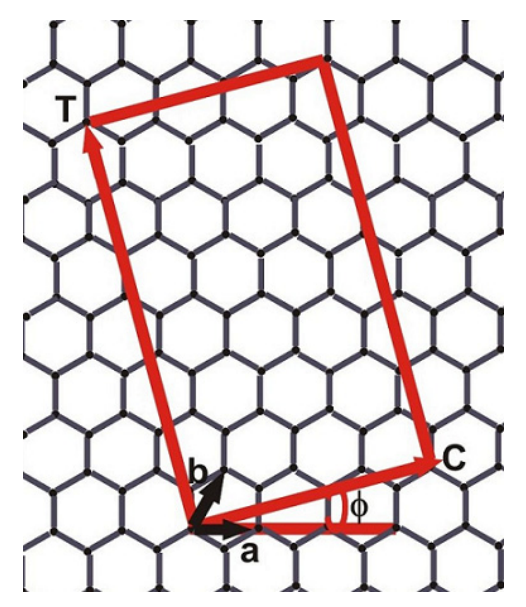

Figure 1: Unrolled hexagonal lattice of a $(3,1)$ single-walled carbon nanotube as a graphene sheet.

Besides the electrical properties, other properties of a CNT that are of interest is its heat transfer capacity and resistance to tensile stress which have also been shown to be dependent on the chirality of SWCNT. Nevertheless, chirality has little effect on the Young's modulus of SWCNT [14].

\subsection{Investigations of mechanical and electrical properties on individual CNTs}

\subsubsection{The main investigating tool}

In particular, the fundamental electrical and mechanical properties of individual SWCNTs generate interest and speculation, and require a detailed understanding. Atomic force microscopy (AFM) or scanning force microscopy (SFM) has been widely used as 
an important tool to measure and understand these properties, because of its ability to scan and image insulating surfaces, its high resolution and high measurement accuracy, and its technical simplicity. Moreover, by employing a conductive tip, AFM can simultaneously determine both the topography and resistivity of MWCNT [15] and SWCNT [16].

\subsubsection{Investigation of radial mechanical properties of SWCNT}

A SWCNT resembles rolling up single graphene sheet into a cylindrical shell, which could be treated as transversally isotropic material, and its elastic modulus and Poisson's ratio weakly depend on chirality when its radius $\left(\mathrm{R}_{\mathrm{cnt}}\right) \geq 0.5 \mathrm{~nm}[17-19]$. With similar definition as Young's modulus, Effective radial modulus : $\mathrm{E}_{\text {radial }}=\frac{\mathrm{F} / \mathrm{A}}{\delta / \mathrm{D}},(\mathrm{F}, \mathrm{A}, \delta$ and $\mathrm{D}$ are the normal force acting on tube, applied area, radial displacement and tube diameter, respectively) has been investigated several times for multi-walled CNT (MWCNT) $[8,20,21]$ and once for SWCNT [22] with nanoindentation without regarding the indentation of the substrate. However, it is difficult to originally locate the vertex of an AFM tip perfectly above the ridge of $\mathrm{CNT}$ as required by nanoindentation (otherwise $\mathrm{F}_{\text {vertical }} \neq \mathrm{F}_{\text {normal }}$ ), sticking to without pressing the sample, and the drifting of cantilever and sample can make it more difficult to repeat this process. Moreover, inevitable contamination and defects from the sample synthesis process in addition to the shape factors of the CNT and substrate surface can give rise to considerable differences in cross-sections and mechanical properties measured at different positions of a CNT [23]. Understanding of the effective elastic properties of CNT in radial direction is essential for its use in the composite material and integrated circuit system under high 
compressive pressure. In this dissertation, an alternative measure is presented, which combines horizontally aligned SWCNTs sample with AFM to gain better measurement accuracy.

\subsubsection{Investigation of the electrical properties of SWCNT}

For the last 15 years, several groups have investigated the electrical properties of CNTs with AFM equipped with conducting probes. Dai et al. [15] was the first to develop a general approach that combines conventional lithography, to electrically contact single ends of CNTs, and C-AFM to determine the conductivity of individual nanostructures protruding from the global contact while simultaneously recording their structure. The studies demonstrated that individual CNTs with perfect structure have resistivity of an order of magnitude lower than that found before and is attributed to the defects in the nanotube structure. Yao et al. [24] have measured the intrinsic transport properties of metallic SWCNTs under high-field by using low-resistance electrical contacts and measured the current density carrying ability of individual SWCNTs. They have also shown that the optical phonon emission is the dominant scattering mechanism for the dramatic conductance drops at high fields. On the basis of Dai's work, Pablo et al. [25] have developed a technique of AFM to investigate the fundamental transport properties of SWCNTs as a function of their lengths. A nonlinear resistance vs. length behavior discovered in the study shows a non-Ohmic electronic transport through the nanotube, which indicates elastic electronic transport in this one-dimensional system. The intrinsic nonlinear R vs. L dependence for SWCNTs is confirmed by theoretical calculations [26]. During the same time, Pablo's group published another paper [27] where they apply stresses to a SWCNT and find that in the I-V curves, the high-bias 
current characteristics change from saturated before the deformation to non-saturated after it, which suggests that the current saturation of $\mathrm{I}-\mathrm{V}$ curve is because of the intrinsic structural properties of the SWCNT. This proposed reason is confirmed later by Barboza et al. [28] who has experimentally observed the semiconductor-metal transition in SWCNTs induced by AFM tip compression. Besides the radial deformations test, a more sophisticated electromechanical system is constructed by Cao et al. [29] to explore the tensile stretching influence on electrical properties of various types of suspended SWCNTs. Using AFM tip as an electrical probe, Park et al. [30] have calculated the mean-free paths for both low and high bias regimes of the same SWCNT and infer the electron scattering rates which are consistent with calculations for acoustic phonon scattering at low biases and optical phonon scattering at high biases. To continue with Pablo's work, Navarro et al. [31] employed an AFM tip to an electrode and induced radial deformations in the radial electromechanical properties measurement. With this technique, they have directly probed the local electronic structure of carbon nanotubes which are radially deformed. Despite of the tremendous endeavors from the previous researcher, electrical properties of SWCNTs are not conclusive enough for further applications. For instance, even though Pablo et al. [25] and Park et al. [30] have shown that electrical transport in metallic SWCNTs closely resembles ballistic conduction, the unresolved perspective is whether it is the mean free path in metals that can be longer than the size of SWCNTs, or the intrinsic property of SWCNTs itself. To find out the exact artifact, there is a large scope of future research. More fundamental studies are needed both theoretically and experimentally. 


\subsection{Investigation of CNTs in electrochemistry}

In general, carbon materials in various forms have been used extensively as electrodes in analytical electrochemistry for its wide electrochemical potential window in aqueous and organic solution [32] and because of its several important attributes including ready availability, chemical and mechanical stability, its bio compatibility. Carbon nanotubes have outstanding structural and electronic properties compared to conventional carbon materials used in electrochemistry such as glassy carbon (GC), graphite, and carbon fiber $[32,33]$. The advantages of using CNT as electrodes include:

(1) CNTs have molecular size diameters and high aspect ratio.

(2) CNTs are mechanically robust and thermally, electrically and electrochemically stable both in air and in electrolyte.

(3) CNTs have high electrical conductivity and low capacitance.

(4) CNT electrodes often show faster electron transfer kinetics than traditional carbon electrodes.

(5) CNTs show electrocatalytic activity that can reduce overpotential.

In the last decade, numerous CNT based electrochemical sensing mechanisms and devices have been reported [33]. The study [34] on individual single SWCNTs has significantly improved our fundamental understanding of their electrochemical properties. Because of the challenges of sorting different type of CNTs in large scale, applications on the basis of large quantity of CNTs are more feasible for short-term applications. Large quantities of CNTs, in different forms, have been studied for various practical electrochemical sensing applications [8], particularly CNT thin film attracted the most attentions. Numerous methods have been developed to prepare CNT thin films. 
The CNT thin films can be broadly classified into two categories, solution-based deposition and direct growth [21] types. Typical examples are membrane filtration [23] and chemical vapor deposition (CVD). The use of CVD method enables the direct growth of pristine CNT thin film on insulating surfaces, without the need for further purification, owing to the reduced amorphous carbon and metal content. Recent studies have shown that SWCNTs, arranged in a planar, extremely low surface coverage $(<1 \%)$ random network, on an insulating substrate, are extremely promising electrodes for low concentration electrochemical detections $[35,36]$ In addition to random network, horizontally aligned SWCNTs arrays have also been successfully prepared by CVD method. The alignment of CNT arrays can play important roles in the electrical properties of CNT thin film based FET devices [21, 37, 38]. Very recently, enhanced biosensing performance of FET device based on aligned CNT arrays has been reported [39]. The enhancement is attributed to the significantly reduced number of CNT-CNT junctions. However, the electrochemical properties of horizontally aligned SWCNT arrays have not been systematically investigated.

\subsection{Remaining issues of CNT research}

The outstanding physical properties stated above endow CNTs tremendous application potentials and commercial values in various industrial fields. However, while carbon nanotube offers exciting possibilities, there are also challenges that face CNT industry. There are several primary obstacles and challenges for the realization of CNTs' promising technological applications. Up to this point, there is lack of low cost techniques for controlled production of CNT with desirable chirality or composition to mass produce CNT-based nanodevices. Meanwhile, more experimental methods and 
technology are needed to accurately characterize their properties in order to apply CNTs with well-known characteristics in areas such as high accuracy engineering. The objective of this dissertation is to continue investigate the synthesis controllability, as well as the mechanical and electrical/electrochemical properties of single-walled carbon nanotubes on the basis of the previous studies. The result of the investigation can also shed light on the research of MWCNTs, which are viewed as concentrically arranged SWCNTs. The results of my study are expected to help further understand the properties of SWCNTs and develop future SWCNT-based devices. 


\section{Chapter 2}

\section{Review of synthesis methods of carbon nanotubes}

\subsection{Introduction of synthesis methods}

Today, CNT has become a common interest in material science and engineering. However, after more than two decades of continued efforts worldwide, society is still eagerly awaiting for the CNT to find applications in household products. Despite the large number of publications and patents on the synthesis of CNT, insufficient production and uncompetitive cost of CNTs with respect to the prevalent technologies is still the bottleneck that stops CNT from wide application. Therefore, the following research perspectives as mentioned below are of interest:

1) Developing methods for cost effective production of large quantities of CNT.

2) Developing methods for the selective growth of SWCNTs depending on preferred orientation, deposition and electrical properties.

The answer to these problems is a proper understanding of the CNT growth mechanism, which requires more detailed studies to analyze the experimental results. High quality CNTs can be produced by the following synthesis techniques.

\subsubsection{Physical methods}

\section{Arc discharge}

Multi-walled and single-walled [3, 40] carbon nanotubes were discovered in the carbon soot of graphite electrodes during an arc discharge process which was designed to produce fullerenes [2]. In the arc discharge experiment, the carbon element that is contained in the negative electrode sublimes as the consequence of the high-discharge 
temperature. Because CNTs were originally observed with this method, it has been widely used in nanotube synthesis ever since.

In an inert gas atmosphere, a DC electric arc discharge can be ignited by a short contact between a pair of electrodes, which are typically water-cooled graphite rods separated by a few mms. Consequently, electrons are freed from the cathode and an electron cloud is formed. The empty space between the two electrodes resulting from the dissociation from each other is filled up by electrons and by the ambient gas. The accelerated electrons ionize the gas molecules in a cascade-like manner by impact ionization on their way towards the anode. Meanwhile, positive charge carriers are moving towards the negative counter pole. The arc will be ignited as soon as enough charge carriers are accumulated in the conducting channel. At the instant of electrode separation, the voltage source will supply the full voltage. The incident electrons lose their kinetic energy by bombarding the anode, which causes the material to sublime. Meanwhile, the cathode is cooled by the discharge work by the electrons. Obtaining stable discharge plasma is one of the most crucial factors for generating an environment favorable for nanotube growth. As the anode is consumed, it has to be tracked towards the cathode continuously. Therefore, maintaining stability is an arduous aspect of the arc discharge method.

The system for the first reported CNT production was a modified Kratschmer reactor [2]. The synthesis rate of a lab Kratschmer reactor can be more that $100 \mathrm{mg} / \mathrm{min}$. The stability of an electric arc is poor because of its natural motion on the cathode and anode at surface level. Moreover, within a brief span of time lapse of the order of few 
minutes, further instability in the DC arc will be caused by uneven consumption of the anode and build-up of material on the cathode side.

\section{Laser ablation}

Carbon nanotube can be synthesized by using high power laser vaporization of graphite targets in an $\mathrm{Ar}$ atmosphere inside high temperature furnace e.g., $\sim 1200{ }^{\circ} \mathrm{C}$. The nanotube growth using laser ablation has been explained as that attachment of the incoming carbon species at the edges of adjacently growing graphene tubes prolongs the lifetime of the open structure of the tube and results in the formation of a MWCNT.

In 1995, the first use of a laser to ablate a block of pure graphite was reported by Guo et al.[41]. In the ablation process, a pulsed laser is used to vaporize the graphite target in a high-temperature reactor chamber, which is filled with certain inert gas. As the carbon vapor condenses, CNTs start to grow on the cooler surfaces of the reactor. The system can include a cool copper collector to collect the nanotubes. Later on, Guo's group changed the target to graphite mixed with catalytic metal [42], which can be elements or a binary combination of cobalt, copper niobium, nickel, platinum, etc. The composite block is made of a mixture of graphite powder, carbon cement, and the metal, which is next transported onto a cylindrical mold and baked for three hours. The graphite block is mounted inside a furnace with a temperature of approximately $1200{ }^{\circ} \mathrm{C}$ following the solidification of the paste mixture. A laser beam will be pointed at the mixture. Argon gas is pumped along the direction of the laser point. As the laser ablates the target, carbon nanotubes develop and are carried by the gas flow onto a water-cooled surface to be collected. 
The first two methods above are revised versions of physical vapor deposition (PVD), which requires large amounts of energy to evaporate bulk solid carbon in order to yield relatively small volume of product. Moreover, as the synthesis processes for these two methods are difficult to control, the final samples are mainly MWCNT with poor alignment accompanied with different forms of impurities. Therefore, the use arcdischarge and laser ablation was severely limited by all the above mentioned factors as large scale processes for the production of carbon nanotubes.

\subsubsection{Chemical methods}

\section{High pressure carbon monoxide reaction}

In 1999, High pressure carbon monoxide reaction production of CNT was developed at Rice university [43]. The difference between this method and others is that high pressure method uses the gaseous phase catalysts fed in the growth process instead of using the catalyst deposit on the substrate prior to the synthesis. Carbon monoxide gas is often used as hydrocarbon gas to react with iron pentacarbonyl, $\mathrm{Fe}(\mathrm{CO})_{5}$ to form the SWCNT. In this process, the catalyst particles are formed through decomposition of organo-metallic compounds, such as iron pentacarbonyl and ferrocene, under high reacting temperature. The catalyst and hydrocarbon gas are introduced into the furnace simultaneously, then the catalytic reaction will take place in the gas phase. Because the growth time in this process can be manually controlled and the nanotubes grown in this process do not require the substrate support, this method is for mass production of CNTs in large scale. SWCNT has also been successfully produced by reaction between benzene and ferrocene, $\mathrm{Fe}\left(\mathrm{C}_{5} \mathrm{H}_{5}\right)_{2}$, which take place in a hydrogen gas flow [44]. 


\section{Chemical vapor deposition (CVD)}

The Chemical vapor deposition of carbon was reported in early references [45, 46], but it was not until 1993 [47] that carbon nanotubes were formed by this process.

As a continuous process, CVD is an irreversible gas-to-solid deposition process taking place via heterogeneous chemical reactions. Chemical vapor deposition for CNT synthesis thermally decomposes the hydrocarbon precursor such as methane, acetylene and ethanol and deposit carbon atoms on the patterned catalyst on a solid substrate. The important parameters in the CVD synthesis of CNTs include the catalyst, hydrocarbon source, and temperature. Both MWCNT and SWCNT syntheses have been achieved by using CVD method. The formation of tubular carbon solids in $\mathrm{sp}^{2}$ structure is caused by the precipitation of carbon on metallic nanoparticles from the diffused carbon atoms. The further development of CVD methods for CNT synthesis includes the CVD method with ethanol [48] and plasma-enhanced CVD (PECVD) method [49].

Compared to arc and laser methods, as far as crystallinity is concerned, arc- and laser-grown CNTs are superior to the CVD-grown ones. However, although CVD-grown MWCNTs possess inferior crystallinity, the crystallinity of SWCNTs grown by CVD is close to that grown by arc or laser methods. Chemical vapor deposition is a much economically plausible technique with a simple setup that can easily be scaled up to industrial production level. It can produce highly purified CNTs in large quantity under moderate temperature. In addition, the better controlled parameters in this technique allow CNTs to be synthesized into different forms of architectures. CVD is the only means for aligned CNT synthesis in both 2 dimensional and 3 dimensional (see Figure 2). 


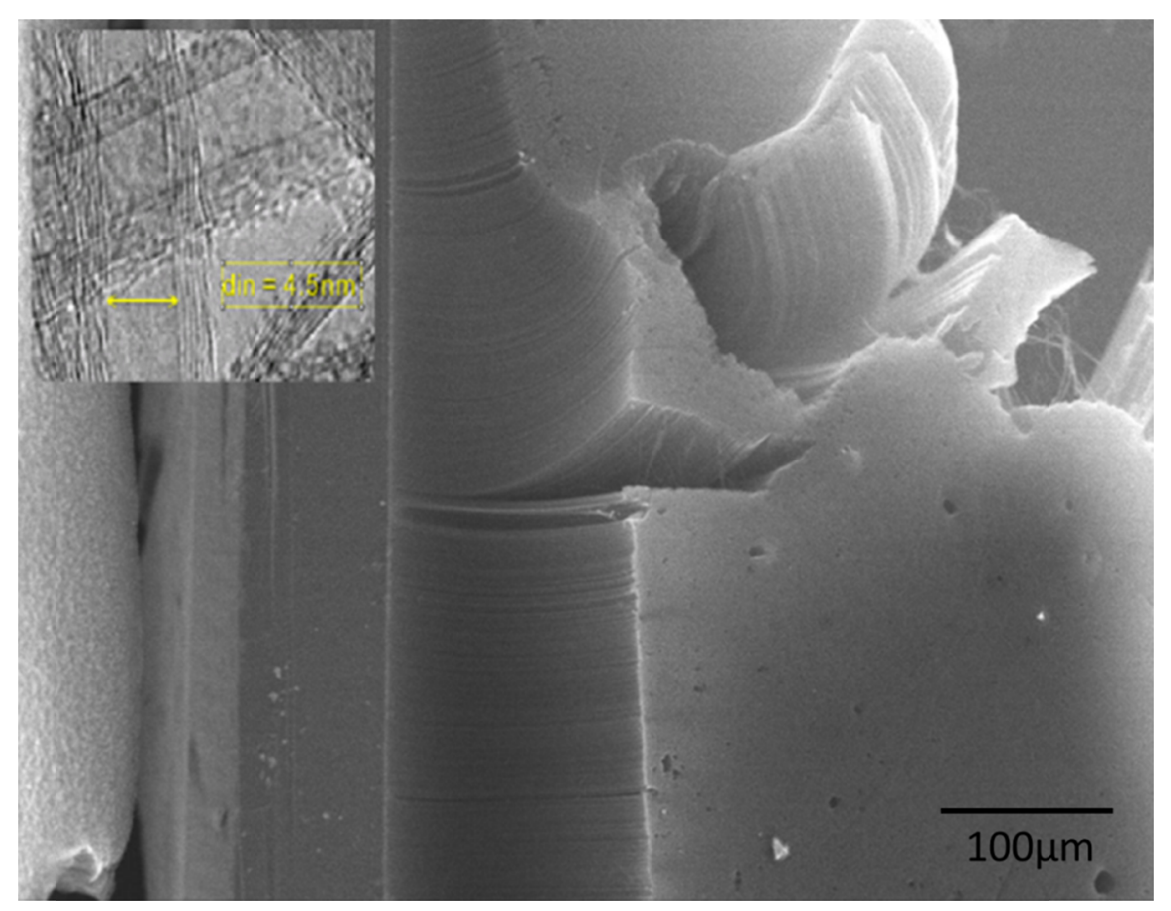

Figure 2: A top view SEM image of synthesized high quality, high density vertically aligned CNTs by our CVD system. Inset: TEM image of individual CNTs on same sample shows inner diameters of $\sim 4.5 \mathrm{~nm}$.

\subsection{Synthesis of single-walled carbon nanotube}

\subsubsection{Synthesis of single-walled carbon nanotubes by arc-discharge}

As the first method that has ever been used for the SWCNT production, Arc discharge involves the evaporation of graphite rods and the following condensation of evaporated gaseous carbon atoms. In the arc discharge process, the carbon atom was evaporated by the plasma of helium gas, which is generated by the high currents between the carbon anode and cathode electrodes. The arc-discharge reactor is shown schematically in Figure 3. During arcing, carbon is deposited at a rate of $1 \mathrm{~mm} / \mathrm{min}$ on the cathode, whereas the anode is consumed [50]. In the production of SWCNTs using this method, three components, i.e., argon, iron and methane, are critical for the SWCNT 
synthesis. Carbon soot produced as a result of arc-discharge precipitated on the iron catalysts elements contained in negative cathode and SWCNTs start to grow. A similar process was carried out by Bethune et al. [40], using thin electrodes with bored holes as anodes. The bored holes in the electrodes were filled with a mixture of pure powder of $\mathrm{Fe}, \mathrm{Ni}$ or $\mathrm{Co}$, which functioned as catalysts. The electrodes were evaporated with a current of 95 - 105 A under an atmosphere of 100 - 500 Torr of Helium environment. SWCNT were also synthesized by the variance of arc-technique by Journet et al. [51]. In the method of Journet et al., the arc was ignited between two graphite electrodes in a reaction chamber under a helium atmosphere of 660 mbar, which resulted in massive production of carbon nanotubes.

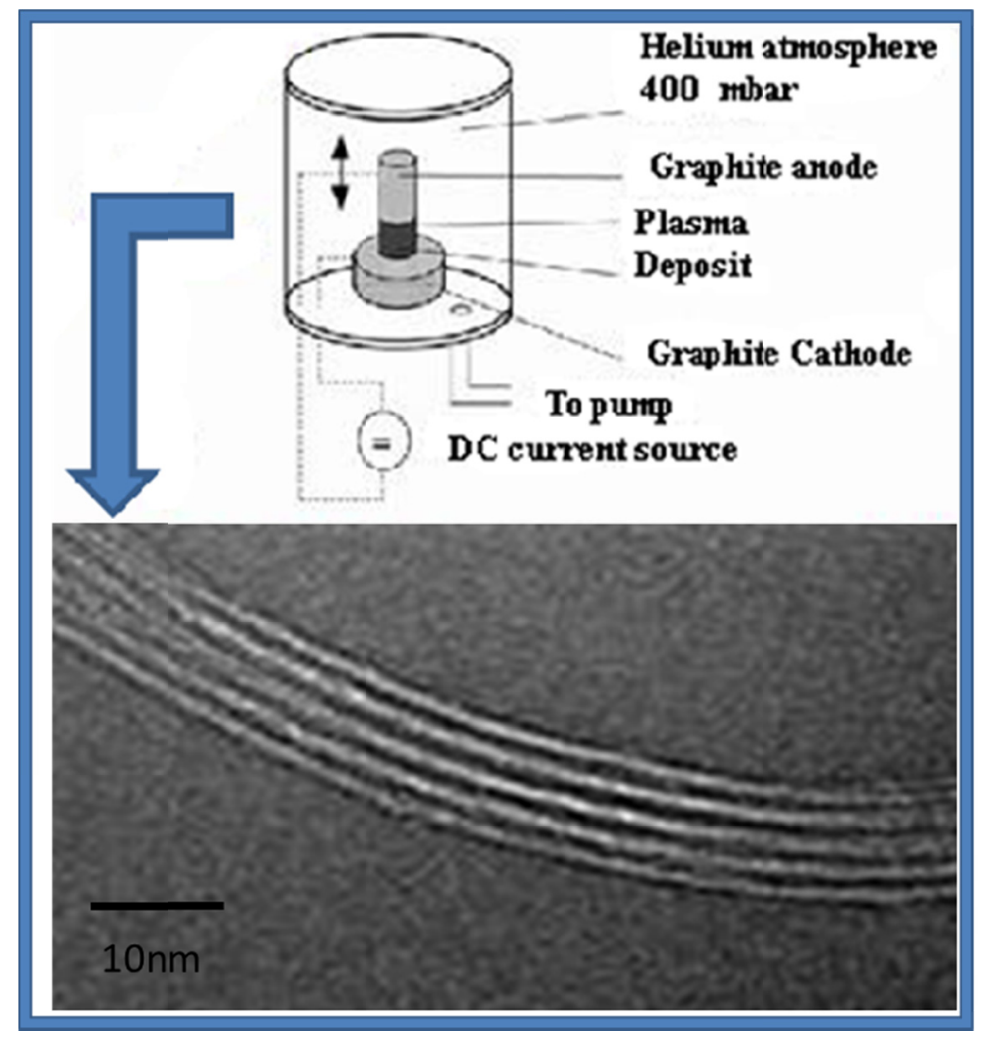

Figure 3: The experimental components of the arc-discharge technique for SWCNT production. 


\subsubsection{Synthesis of single-walled carbon nanotubes by laser ablation}

In a standard setup of the laser ablation system for the SWCNT growth, a oneinch quartz tube is placed in a tube furnace which is operated at a temperature of $1200^{\circ} \mathrm{C}$. The quartz tube is sealed and vacuumed by a vacuum system. A Brewster window plated by anti-reflex layer is used for the incoming laser beam. The graphite target consisting of metallic catalysts is positioned in the center of the furnace to ensure that the ablation process is homogeneous. Usually, the beam is focused to a 3-8 $\mathrm{mm}$ diameter spot on the target. At the upstream side of the tube, the inert gas are introduced into the system, whereas, a mass flow controller and a pressure controller are used at the downstream to control the flow dynamics in the system. The resulted SWCNTs will be carried by the gas flow to a water cooled copper collector to be collected. Usually, the collector is mounted into the quartz tube and located outside the furnace. The schematic illustration is given in the Figure 4. Most of the produced SWCNTs were swept out of the furnace zone by the carrier gas and deposited on a water-cooled copper rod as soot.

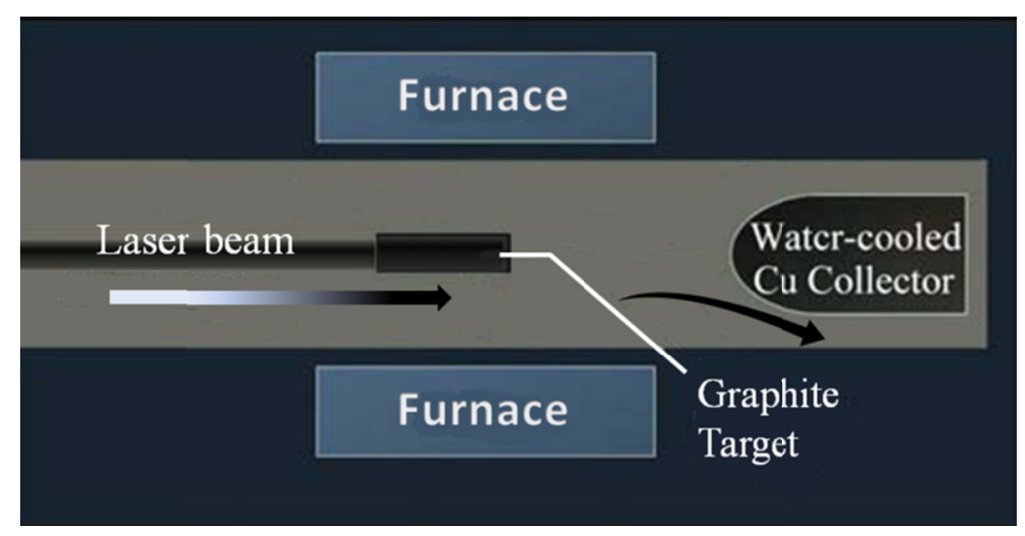

Figure 4: The schematics of the experimental set-up for laser ablation. 


\subsubsection{Synthesis of single-walled carbon nanotube by chemical vapor deposition}

Because of its simplicity and scalability, CVD is most widely used routes among all methods for SWCNT synthesis [52]. While other methods need multiple steps of preparation to produce SWCNTs, CVD can finish it with one single step and provide the sample that is long, clean, defect-free, and even well-aligned [53-55]. Additionally, CVD provide controllability over the spacing, length and orientation of the produced SWCNT sample which have firm electrical and physical contact with the substrate.

The CVD reactor in the FIU laboratory is schematically illustrated in Figure 5. The precursor in the bubbler is ethanol carried into the reactor by gas mixture (often $\mathrm{H}_{2}$ and Ar) in order to control the carbon concentration in the gaseous phase. The chamber for the CVD process is a quartz tube resistor furnace from Thermolyne. The substrate with patterned catalyst on is accommodated by a sample holder that is placed in the center of the thermal furnace.

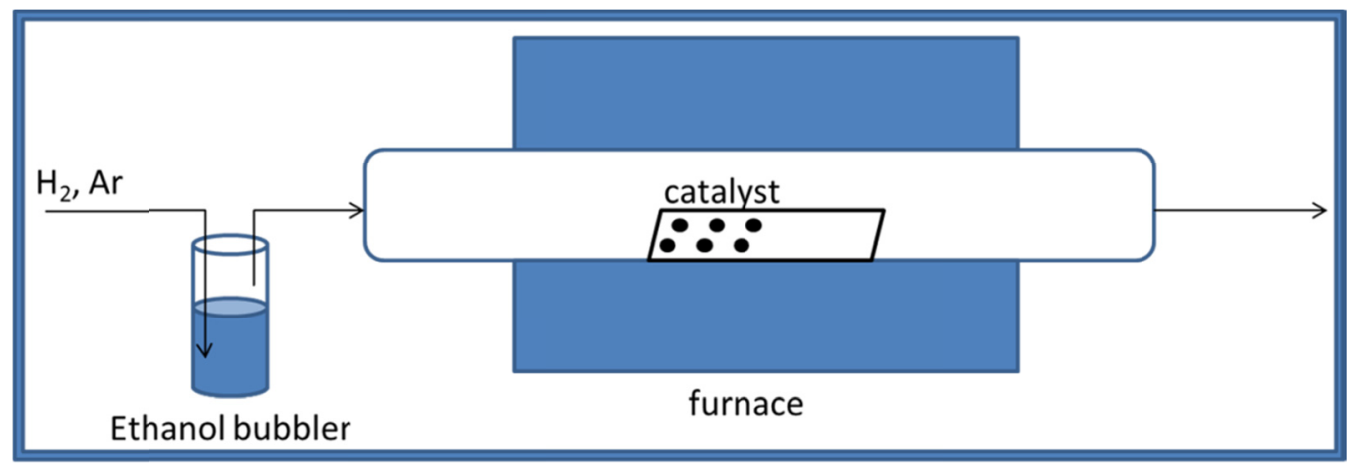

Figure 5: The schematics of experimental set-up of our chemical vapor deposition system.

The CVD methods have been developed and improved significantly over the last two decades, however, the accurately controlled chirality have never been able to be achieved by the traditional approaches using metal catalyst particles. Recently, a generic method to fabricate carbon nanotubes with desired chiralities by employing purified 
single-chirality nanotubes as seeds were developed by Liu et al. [56]. The method typically involves non - involvement of any metal catalyst which by far resembles the vapor phase epitaxy, which finds its common application in semiconductor films synthesis. In their studies, $(7,6),(6,5)$ and $(7,7)$ SWCNTs were successfully Raman spectroscopy indicated unambiguously that this approach bolstered the very maintenance of original chiralities of the nanotube seeds in the produced SWCNTs. Although this cloning process opened up a possibility of chirality control for individual SWCNTs, further research is still in need for the improvement of controllability and selectiveness in large scale synthesis over the chirality, and orientation/alignment of the sample.

\subsection{Alignment control of SWCNTs on the substrate surface}

The salient need for horizontally aligned SWCNTs is bestowed in their ability to provide various devices like FETs and interconnects. Furthermore, when desiring high frequency applications to facilitate high magnitudes of current [57], closed packed arrays of SWCNT's response serves unquestionably the very best to the desired purpose. The challenges that are faced during the synthesis are putting the original pre-existing structures at jeopardy as a result of being subjected to very high temperatures, and the lack of well supported claim of the existence of a reliable method to produce all semiconducting SWCNTs. Hence for a complete implementation of the SWCNT based electronics [58], the aforesaid problems are to be overcome, therefore the study of synthesis of well aligned SWCNT arrays on desired application-based substrate for the electronics industry is one of the vantage areas to be considered [59].

The CVD has proven to be one of the most promising methods of producing well aligned SWCNTs amongst all other methods tried by researchers so far [59-61]. 


\subsubsection{Control of SWCNTs alignment by external electrical fields}

The use of external electric field to orient CVD grown SWCNTs was shown to be possible by Zhang and his coworkers in 2001 [62]. The established electric field was analogous to three capacitors in series formed by the polycrystalline-Si trenches. As the bias voltage was increased, the degree of alignment was enhanced, which was verified by the fact that if no bias voltage was provided, the tubes grew at random between the trenches. A very interesting claim was made in the publication by Ural et al. [63]. The aligned SWCNTs grown between Mo electrodes at a $10 \mu \mathrm{m}$ spacing with an applied electric field suggested that tubes grown from the catalytic region at the initial stages grow into the air in a direction normal to the metal electrode surface, which is the direction of the localized electric field. As the growth continues, the SWCNTs will follow the gradient strength of the electric field. The directional growth control under various growing conditions was also studied by Chiu et al. In their study, SWCNT grown between metallic electrodes had a directional growth control by the use of external electric field with the additional localized control being achieved by using two triangular electrodes with sharp tips in opposition to each other [64]. Another study has used the local built in electric field induced during plasma enhanced CVD [62]. With the help of right choice of materials and geometries forming the microstructure on the substrate surface, the built in electric field could be provided for which was horizontal in direction. The study revealed that the tubes were aligned in a horizontal manner near the surface of the sidewall microstructure and were vertical or inclined as they go far away. 


\subsubsection{Control of SWCNTs alignment by gas flow}

Huang et al. in 2004 [65] were the first to introduce the directional gas flow to orient the growth of SWCNTs. In this method an initial rapid heating is applied on silicon substrate and the deposited catalyst before the carbon feedstock is introduced. $4 \mathrm{~cm}$ long SWCNT were reported to have been successfully grown by Zheng et al at the rate of 11 $\mu \mathrm{ms}^{-1}$ by CVD process with the use of $\mathrm{FE}$ as the catalyst and low gas flow rates of $\mathrm{Ar}, \mathrm{H}_{2}$ and ethanol mixture [66].
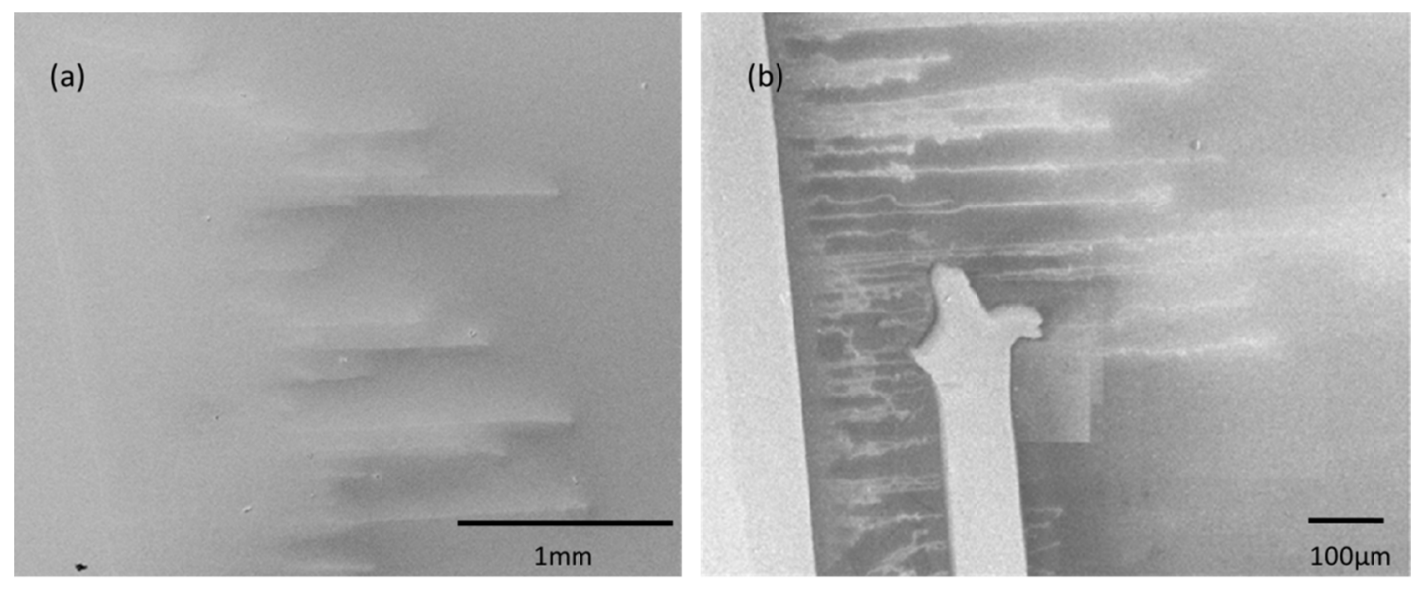

Figure 6: Flow orientated millimeter-long SWCNTs. (a) A pristine sample. (b) A sample patterned with gold electrodes.

On the base of Zheng's experiments, a synthesis module has also been developed in the nanophysics Lab at Florida international University (FIU) for the aligned SWCNTs by gas flow direction control and obtained millimeter long growths. We need to use $90 \mathrm{~nm} \mathrm{SiO}_{2}$ on a silicon as the substrate. Cobalt acetate was blended in the toluene solution with PS-P4VP polymer to form catalyst particle with uniform size. The catalyst was enclosed within the polymer was then treated with oxygen plasma (High) for 20 minutes. The substrate was placed in the 1 inch quartz tube at $504{ }^{\circ} \mathrm{C}$ and ramped the furnace to $900{ }^{\circ} \mathrm{C}$ (ramping rate $18{ }^{\circ} \mathrm{C} / \mathrm{min}$ ) with $300 \mathrm{sccm} \mathrm{Ar}+110 \mathrm{sccm} \mathrm{H} 2$. The CVD 
growth was then carried on in the furnace with $300 \mathrm{sccm} A r+30 \mathrm{sccm} \mathrm{H} 2$ bubbling through ethanol at $0{ }^{\circ} \mathrm{C}$ for 30 minutes. The result obtained by my research group at FIU is shown in Figure 6. The Figure 6(a) is blurry because the $\mathrm{SiO}_{2}$ substrate were severely charged by the electron beam, while in Figure 6(b) the charge on the substrate were conducted away by the patterned gold electrode for the electrical transport measurement. To be noticed, the diameters of SWCNTs in the SEM images appeared to be much larger than their real diameters as the consequence of the charging effect.

\subsubsection{Control of SWCNTs alignment by the lattice orientation of substrate}

Selectively cut single crystal substrate such as ST-cut quartz is an alternative crystallographic orientation route for the growth of horizontally aligned SWCNT. The lattice structure of the substrate surface is the responsible factor rather than the steps on the surface. The lattice orientated approach enables the growth of long horizontally aligned SWCNT over a range of growth conditions.

Large scale yield of aligned arrays of SWCNTS of high density $\left(>10\right.$ tubes $\left.\mu \mathrm{m}^{-1}\right)$ with diameters in the range of $1 \pm 0.5 \mathrm{~nm}$ were found to have been the resultant growth on AT-cut and Y-cut-based single crystal quartz substrates [57]. The density of the alignment could be further improved by thermally annealing the ST-cut quartz at $900^{\circ} \mathrm{C}$ for 8 hours before the CVD process and by evaporation of the sub-monolayer films of iron that serves as high density catalyst stripes on the surface and the carbon feed gas being ethanol [67].

The horizontally aligned semiconducting SWCNTs on quartz wafers were synthesized in high density by Liu and his group at Duke in 2009. The thin film transistors which were derived from the synthesized sample had the capacities to provide 
high levels of on-driving current density of the magnitude of $220 \mu \mathrm{A} / \mu \mathrm{m}^{2}$. Their systematic studies also led to the proposal of the fact that the high selective growth is the result of the etching effect and chemical reactivity difference of metallic and semiconducting nanotubes. Three salient rules could be summarized for achieving highly selective growth of semiconducting nanotubes after very careful scrutiny of the relationship between wafer concentration, carbon feeding rate and percentage of semiconducting nanotubes in the SWCNT arrays that yield out of the process. The three rules that were arrived at could also be used for random SWCNT network synthesis on silicon wafers. The understanding of these laws is believed to lead us to overcome the problem: the coexistence of metallic and semiconducting nanotubes for the various synthesis methods.

Epitaxial growth mechanism of SWCNTs on ST-cut quartz has been reported from X-ray diffraction (XRD) and AFM studies that were conducted by Rutkowska et al [68]. The allotropy of the single crystal quartz varies with temperature, i.e., the $\alpha$-phase being the form at room temperature and the $\beta$-phase at higher temperature, which is the condition at which the growth takes place. The indication of epitaxial growth was confirmed by measuring the angle of the bent tubes that were close to the values of Si-OSi.

Xiao and co-workers [69] did a theoretical analysis of the preferential growth of SWCNTs on single crystal quartz substrates that had different crystal directions. Simulations of molecular mechanics that were performed on the basis of inter-atomic potentials on analysis led to the confirmation that excellent alignment is possible with one dominant direction for Y-cut and ST-cut substrates. 
My own experiments for lattice orientated SWCNT synthesis were carried out with similar growth conditions as that of Kocabas et al in 2007. except for using cobalt as the primary catalyst choice. The methodology and result of this research were discussed in details in Chapter 3. 


\section{CHAPTER 3}

\section{Synthesis and characterization of single-walled carbon nanotubes in this research}

\subsection{Synthesis method}

As far as the SWCNT is concerned, CVD is a versatile mean that offers harnessing of various hydrocarbons in different states (solid, liquid or gas), enables the use of different substrates, and allows SWCNT growth on predefined sites of patterned substrates and in a variety of forms, such as powder, films, straight or coiled, aligned or entangled nanotubes. Compared to the arc and the laser methods, CVD method is superior for the yield and the purity. Above all, CVD is the only answer so far when it comes to structural control or nanotube architecture.

In our lab, the horizontally aligned SWCNTs were successfully synthesized on a St-cut quartz substrate by CVD. The properties of the sample has been previously studied in detail both experimentally $[61,70]$ and theoretically [71] by other research groups. It is suggested that interaction between the SWCNTs and the quartz lattice leads to the selective growth of aligned semiconducting nanotubes. However, until present, the degree of control over the synthesis of SWCNTs is still limited. It has been tried in this dissertation to gain a better control over the morphologies and properties of the synthesized SWCNTs by adjusting the pre-growth and in-situ conditions.

The lattice-oriented alignment properties of the SWCNTs grown on St-cut quartz have been studied in detail (See Chapter 2). The schematic sketch of our CVD system is given in Fig. 2.4. 0.5mm thick St-cut single-crystal quartz substrate (Hoffman Materials, Inc., single side polished; TI Corp. double side polished) was used to the SWCNT growth. A strip of cobalt catalyst (about $2.5 \mathrm{~mm}$ in width and $0.2 \mathrm{~nm}$ in thickness) was 
patterned using a shadow mask and deposited by an electron-beam evaporator (EGN4, Oxford Applied Research) onto the substrate. The strip was oriented perpendicular to the $\mathrm{X}$ axis of the quartz crystal which corresponds to the growth direction of the aligned SWCNTs. Nanotubes were grown by CVD method using ethanol as carbon source at 925 ${ }^{\circ} \mathrm{C}$ in $50 \mathrm{sccm} \mathrm{Ar}$ and $50 \mathrm{sccm} \mathrm{H}_{2}$ flow for $12-15 \mathrm{~min}$. The salient characteristics of the CNT thin film under the same growth condition are quite reproducible.

\subsection{Characterization of single-walled carbon nanotubes}

\subsubsection{Image profiling and analysis}

The as-grown SWCNT arrays on single crystal quartz substrate were imaged by SEM. One typical image is shown in Figure 7(a), which was taken at the position with a distance of $\sim 30 \mu \mathrm{m}$ from the boundary of the catalyst region. The distribution of CNT becomes quite uniform in this region. In the image, most CNTs orientate in parallel from top to bottom, which is the preferred direction guided by the quartz substrate. However, quite a few CNTs also grow in random directions on the substrate. By calculating the ratio between the number of aligned CNTs to the total number of CNTs in a selected area (i.e., a $50 \times 50 \mu \mathrm{m}$ square region), the ratio of alignment can estimated to be about $88 \%$. The length of majority of CNTs is between $20 \mu \mathrm{m}-100 \mu \mathrm{m}$. The area density of the aligned CNT can also be roughly estimated from the SEM images by counting the number of

CNTs in a $10 \mu \mathrm{m} \times 10 \mu \mathrm{m}$ rectangle, which is about $0.5-3.0 \mathrm{CNTs} / \mu \mathrm{m}^{2}$. Similar morphologies of SWCNT arrays have been reported by Roger's group [57]. 

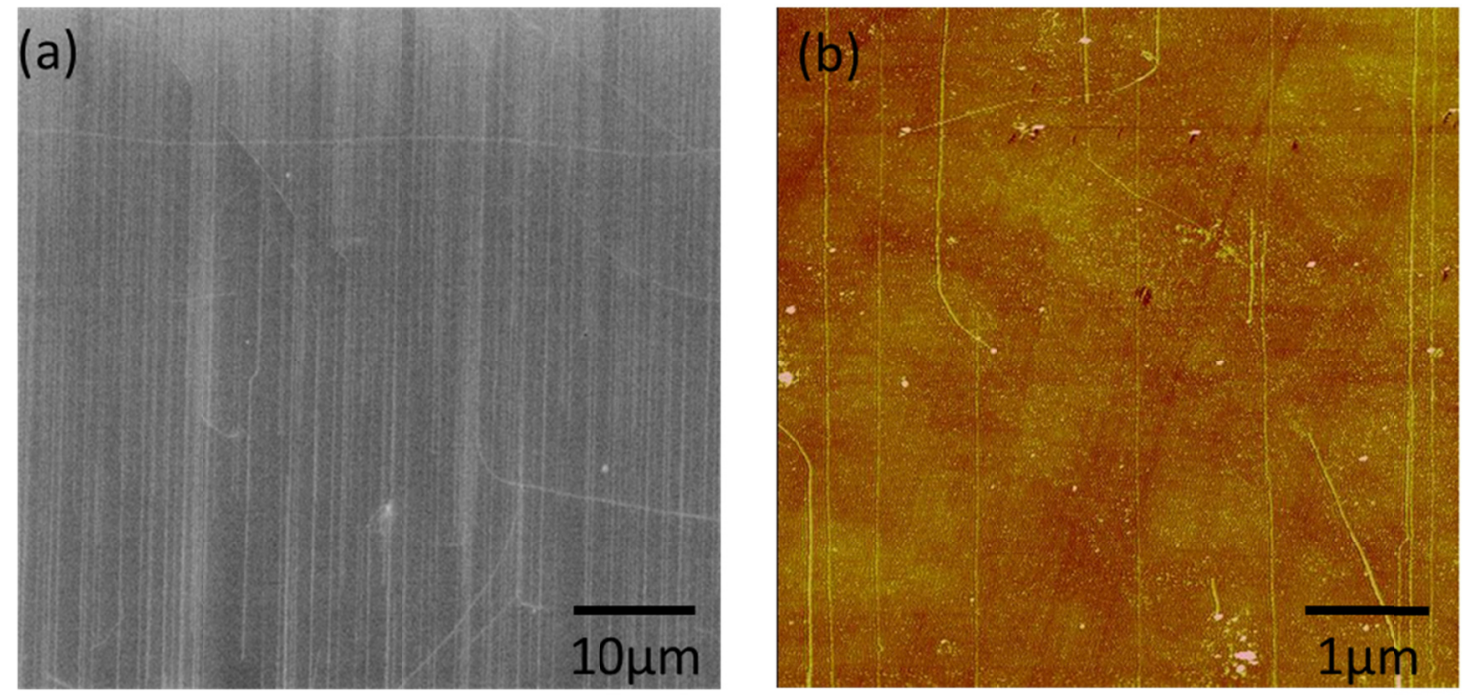

Figure 7: (a) A typical SEM image of as-grown SWCNTs. (b) A topographic AFM image of my sample.

I also used atomic force microscopy (Multimode 5, Bruker) to image the CNT (Figure 7(b)) in roughly the same area as the SEM image. Both aligned and misaligned CNTs were visible. Therefore, a sub-monolayer film of CNTs was formed on the quartz substrate through the connections between the aligned and misaligned CNTs. The average diameter of the CNT was acquired from the height profile (Figure 8(b)) of the tapping-mode AFM images. Taking advantage of the perfectly straight SWCNT sample, the diameter can be measured by taking the average of the height profile of a contamination free portion of an individual SWCNT along its length direction (see Figure 8(a)). The data collection method contributed significantly to the radial elasticity measurement described in Chapter 4. As shown in Figure 8(c), an average outer diameter of $1.4 \pm 0.3 \mathrm{~nm}$ was determined by using a Gaussian fit to the CNT diameter distribution histogram. The diameter distribution histogram suggests the majority of the as-grown CNTs are SWCNTs, which is consistent with the previously reported results using the 
similar growth method $[57,72,73]$. Calculated from the area density and CNT average diameter, the surface coverage of the partially aligned CNT thin film is about $0.1-0.6 \%$. Therefore this CNT thin film is ultrathin and a sub-monolayer.
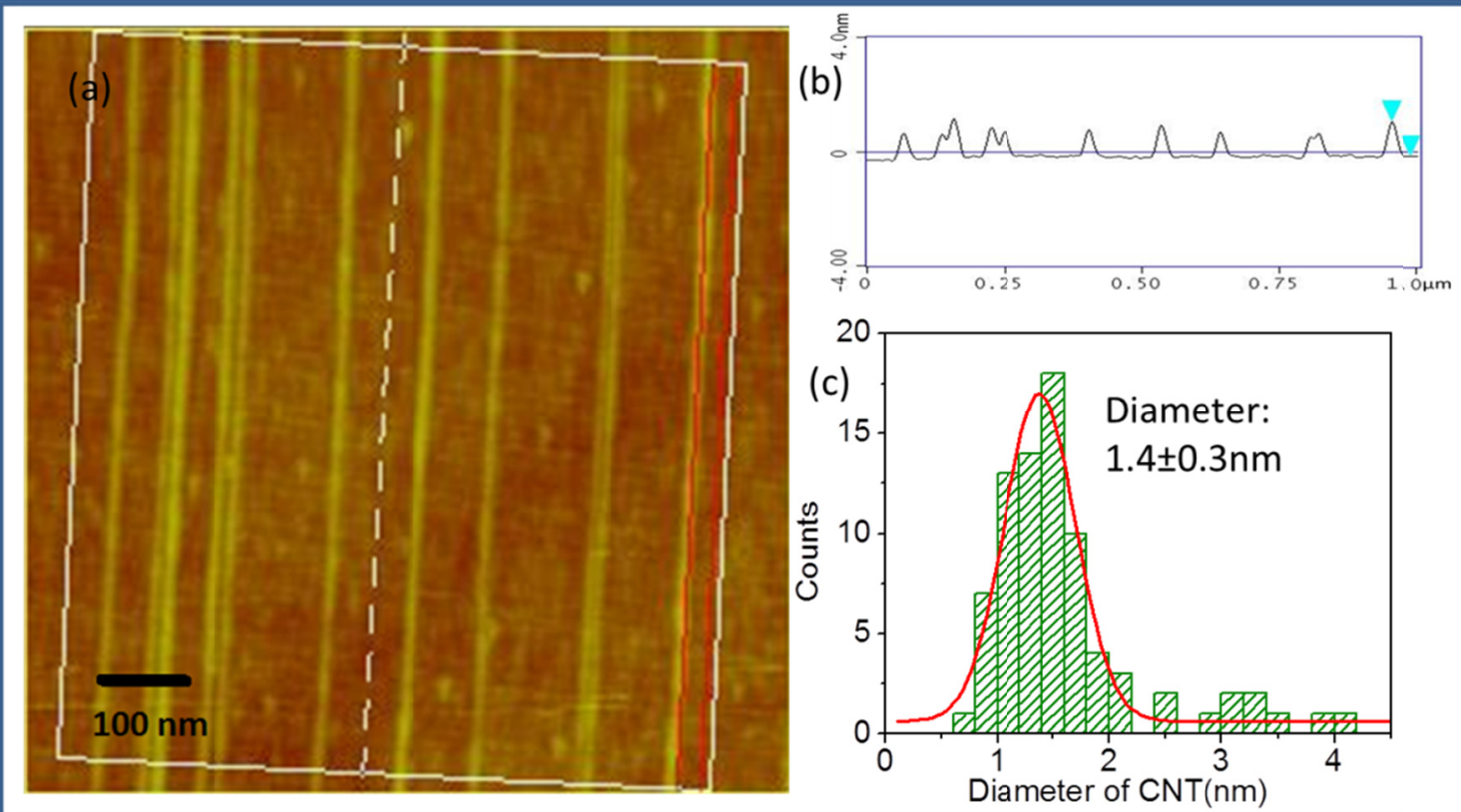

Figure 8: (a) Average diameter measurement over the length direction of the individual SWCNTs in the AFM image. (b) The average height profiles of the SWCNTs in the image. (c) The diameter distribution of 80 SWCNTs.

\subsubsection{The effect of annealing St-cut quartz on SWCNT synthesis}

The alignment of the grown tube is affected by the thermal annealing in air as the result of the morphological effect it has on the single crystal quartz substrates. Some of the conditions of thermal annealing in air are at $950{ }^{\circ} \mathrm{C}$ for a duration of $1.5 \mathrm{~h}$, [71] at $900{ }^{\circ} \mathrm{C}$ for a duration 30 minutes [68] at $900{ }^{\circ} \mathrm{C}$ for $8 \mathrm{~h},[74]$ and $750{ }^{\circ} \mathrm{C}$ for $15 \mathrm{~h}$. [75]. The effects of the annealing process of St-cut quartz leading to roughness in the yield of SWCNT were investigated in details by Ibrahim et al [75]. The outcome of their study led to the conclusion that thermal annealing is not a required step in achieving an aligned 
growth of nanotubes, but the benefit lies in enhancing the tubes' density and length. The positive effects of thermal annealing of St-cut quartz in air was also reported by Rutkowska et al. [68].
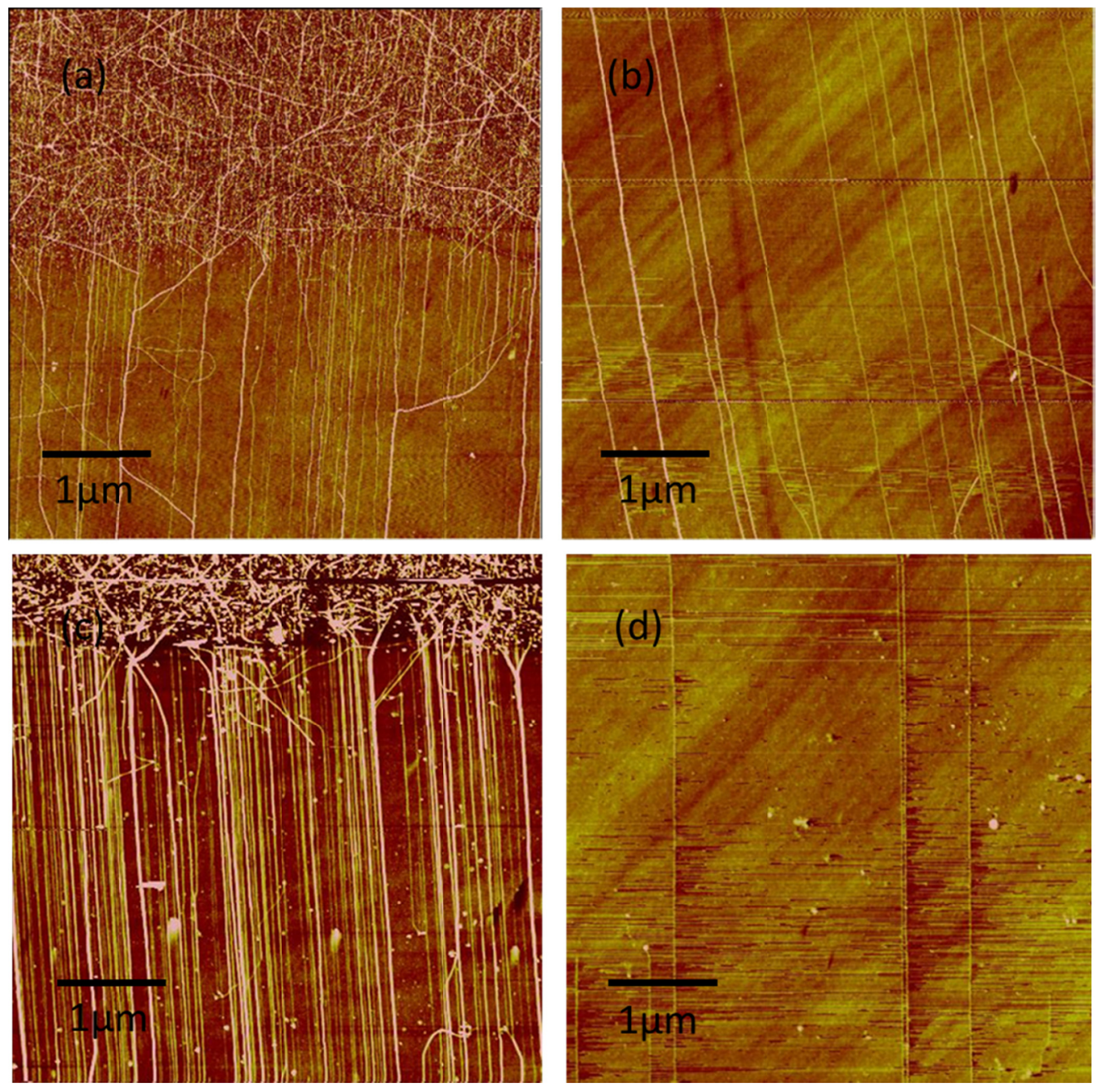

Figure 9: (a) SWCNTs synthesized on original St-cut quartz without pre-growth annealing. (b) SWCNTs synthesized on St-cut quartz annealed for 4 hours (c) 8 hours, and (d) 20 hours.

In our study, thermal annealing has been carried out for $4 \mathrm{~h}, 8 \mathrm{~h}$, and $20 \mathrm{~h}$ at 950 ${ }^{\circ} \mathrm{C}$. The conclusion regarding the density and length of aligned SWCNTs is consistent with that from Ibrahim et al. (2011). Aside from the density and length of the tubes, it is also found that the degree of alignment was affected by the thermal annealing at this high 
temperature. In Figure 9, the statistical survey of straightness of individual SWCNT showed that the alignment was improved by annealing the substrate for $4 \mathrm{~h}$, and was significantly enhanced when the annealing time was more than 8 hours. It was also clearly shown in both Figure 9 (a) and (c) that in the areas of the patterned sites aligned tubes were obtained but in the areas over the non-patterned catalyst region random network of tubes were produced. As the cobalt catalysts were patterned at specific locations on the ST-cut quartz substrate surface before the commencement of the CVD reaction, it was inferred that the alignment was a result of the surface lattice structure of the selective cutting of the substrates.

In some cases, long thermal annealing generated steps spaced sufficiently far apart to allow imaging by AFM. The surface structure in Figure 10 (a) and (b) was observed on the substrate surface after the 20 hours thermal annealing at $950{ }^{\circ} \mathrm{C}$. Similar steps as the consequence of thermal annealing cited by Kocabas et al. (2007) are in the range of 0.7 to $1 \mathrm{~mm}$ high with the spacing in the range of 30 to $35 \mathrm{~nm}$. Annealing forces result in a reduction of stability of atomic steps to collectively gather the faceted nanosteps thereby reducing the surface energy [76]. A consistency could be observed at a comparative level with Ibrahim et al. (2011), whereby the surface roughness reduced significantly after annealing for $15 \mathrm{~h}$. But beyond this time duration the surface becomes rough again. It is believed that the guidance process is jeopardized by the surface structural defects [75]. 

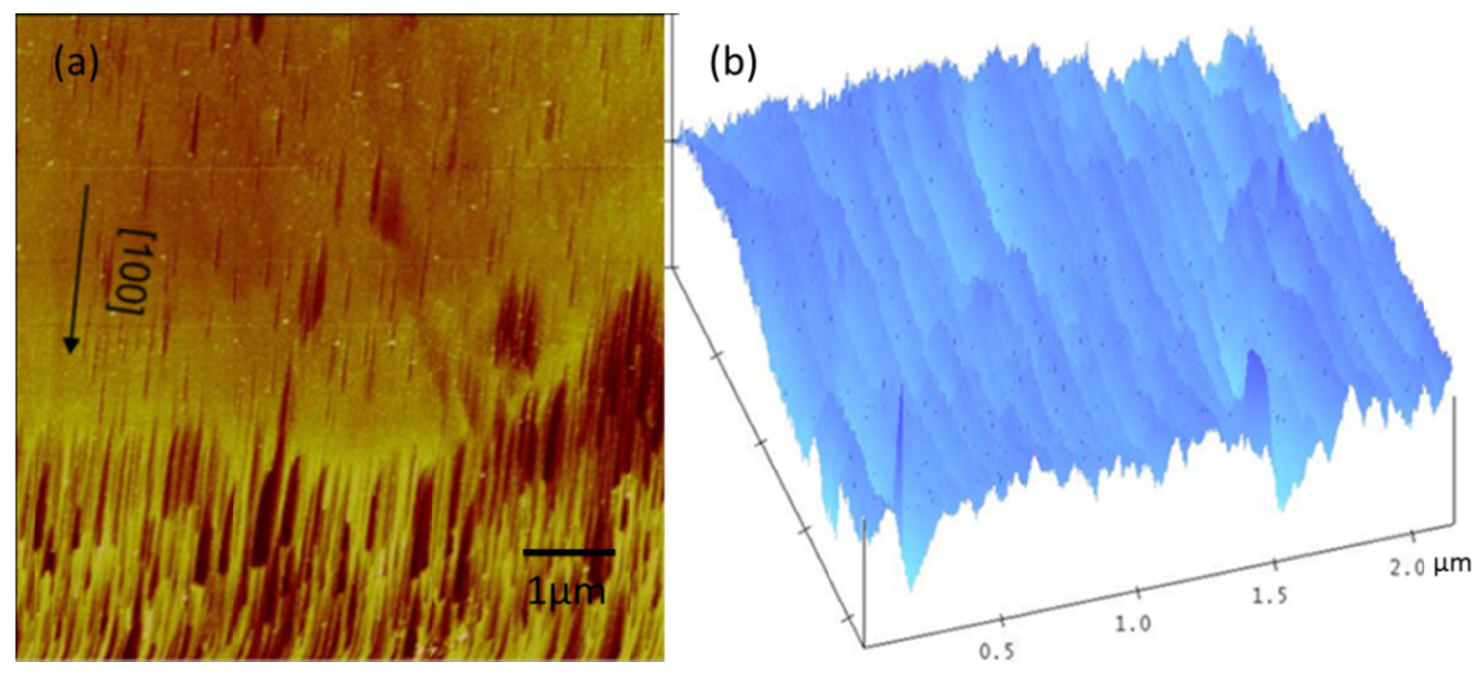

Figure 10: (a) An AFM image of step features along the [100] direction on St-cut quartz surface after 20 hours thermal annealing. (b) A 3-D AFM image of the same step features.

\subsubsection{The growth of SWCNTs and the migration of catalyst particles}

A series of 6 high-resolution AFM images $(8 \mu \mathrm{m} \times 8 \mu \mathrm{m})$ were taken to study the density and length distribution of my samples. From Figure 11 (a) to (f), the sample surface was imaged consecutively along the [100] direction, which is the preferred growth direction of the SWCNT. The quartz substrate used for this sample was annealed for 8 hours under the temperature of $950{ }^{\circ} \mathrm{C}$, suggested by the sample characterization and references. On the edge of the catalyst band, 162 SWCNTs were captured in the image of Figure 11 (a) growing out of the catalyst area. However, only 2 of the SWCNTs have grown more than $40 \mu \mathrm{m}$. 



Figure 11: From image (a) to (f), a continuous imaging process of $48 \mu \mathrm{m}$ along the growth direction of sample 1.

The density vs. distance from the catalyst region is given in Figure 12. In this region where is close to the catalyst band, the density of SWCNTs decreases exponentially over the growth direction. The length distribution by percentage was derived from Figure 12 and given in Table 3.1.

However, it was noticed that the in Figure 11 (d), (e) and (f) that SWCNTs started to grow at the non-catalyst area, which indicates the drifting of catalyst particles along the preferred growth direction, i.e., the [100] direction on the St-cut quartz surface. This additional growth resulted in a relatively uniform density distribution of SWCNTs (1.1 SWCNTs/ from image Figure 11 (d) to (f). 


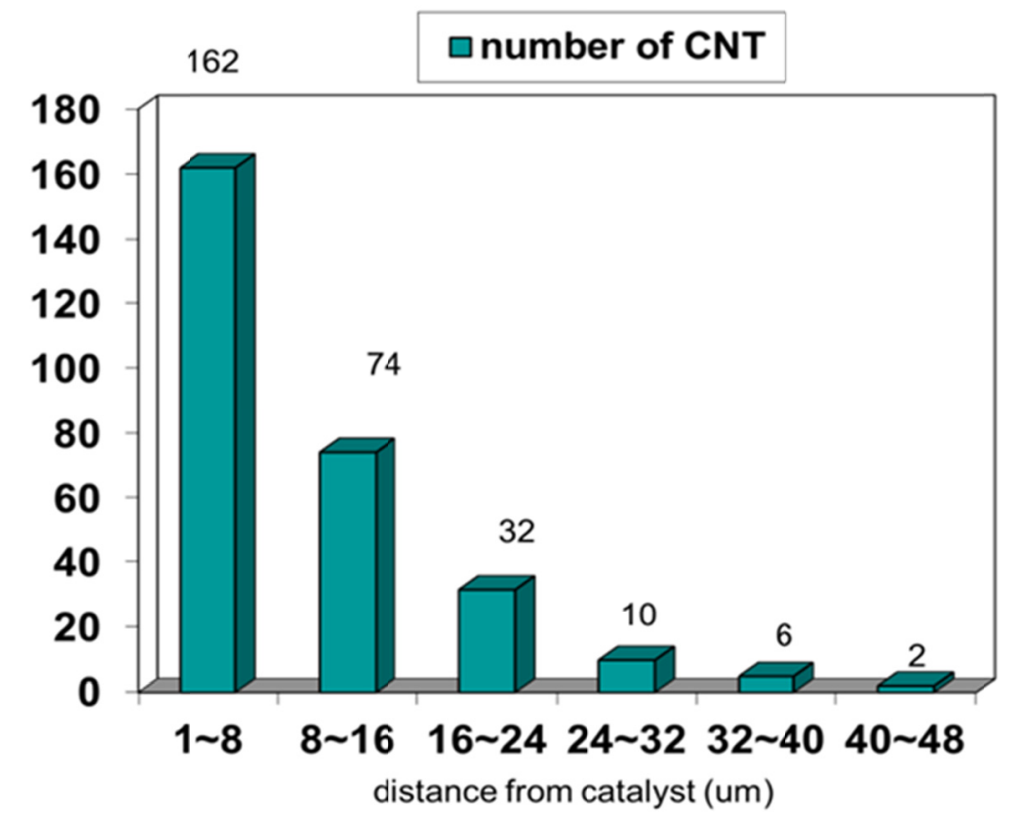

Figure 12: From image (a) to (f) in Fig. 11, the number of nanotubes declines exponentially from 162 to 2 along the growth direction of sample 1.

\begin{tabular}{|l|l|l|l|l|l|l|}
\hline Length $(\mu \mathrm{m})$ & 1 to 8 & 8 to 16 & 16 to 24 & 24 to 32 & 32 to 40 & $>40$ \\
\hline percentage & $54 \%$ & $26 \%$ & $14 \%$ & $2.5 \%$ & $2.5 \%$ & $1.2 \%$ \\
\hline
\end{tabular}

Table 1: The length distribution of SWCNTs on sample 1.

The migration of the cobalt catalyst particle along the growing direction was also observed in other samples (e.g., sample 2) in the area some millimeters away from the catalyst particle. Again, shown in Figure 13, on sample 2 with a higher SWCNT density, a series of AFM images $(6 \mu \mathrm{m} \times 6 \mu \mathrm{m})$ were taken $2 \mathrm{~mm}$ away from the catalyst band along the SWCNT growing direction. A number of SWCNT endings can be seen along the 24 $\mu \mathrm{m}$ long image. However, the number of SWCNTs does not show an apparent declining pattern within the image series like it does in the image series in Figure 11. In the region where is far away from the patterned catalyst band, a dominant fraction of SWCNTs 
grows from the migrated catalyst particles, which have a relatively uniform distribution on the substrate surface. These uniformly distributed catalyst particles further yield a relatively uniform distribution of SWCNTs, which provides an ideal platform for the electrochemistry studies with my pristine sample (See chapter 5). The migration of catalyst particles was then supported evidently by the serpentine SWCNTs and the nanobarrier phenomena, both of which were discovered on the samples used in this research.



Figure 13: From image (a) to (d), a continuous imaging process of $24 \mu \mathrm{m}$ along the growth direction of sample 2.

\subsubsection{Serpentine carbon nanotubes}

As referred to by many other researchers, the serpentine CNTs were also found in the SEM characterization process of the sample. A two-step mechanism for the serpentines was proposed by Geblinger and his coworkers [69]. The features of the proposed mechanism included primarily the guided growth of the tubes by the directed gas flow amidst the tip of the tube and the catalyst particle migrating on the substrate surface. As the tube achieves the critical length a fall process, which is a rapidly oscillating by nature, is introduced which is the consequence of a race between the SWCNT substrate adhesion and the flow induced tension. The result of the zipping motion leads to a pull trying to move the tube away from the center of mass and a drag motion is evolved in the upper region in an opposite manner leading to the induction of a 
loop tangent to the surface of the step. A $U$ shape is produced as the consequence of the changes in the falling direction arising from a large dragging force enough to derail the nanotube. It is believed that the serpentine is evolved by carrying this above mentioned oscillatory process repetitively. In Figure 14 (a), the serpentine nanotubes were observed in the SEM image of the pristine sample. Similar results were also found in another report [77]. Once again, the diameters of SWCNTs in the SEM images appeared to be much larger than their real diameters as the consequence of the charging effect.

\subsubsection{CNT nano-barriers}

The growth of SWCNTs can be interrupted on the quartz surface by using other CNTs, which are directed by the gas flow. Thus by using these CNTs as nano-barriers, SWCNTs grown on the quartz surface via the lattice oriented growth mode can be terminated.

The experimental result is shown in Figure 14 (b), the nano-barrier nanotubes have been pre-grown on the quartz surface by gas flow orientated growth, and have been burned down into small segments in the oxygen environment heated to $500{ }^{\circ} \mathrm{C}$. The segments have successfully blocked growth of a majority of those lattice orientated SWCNTs, with an exception for a few with large diameters. One typical nano-barrier segment was imaged by AFM shown in Figure 14 (c). The same diameter effect has been observed by Feng et al. [78]. Since the nano-barriers also prevented the migration of the catalyst particles from the side of the catalyst band, clear pathways were created on the other side of the nano-barrier without the additional SWCNT growth. 

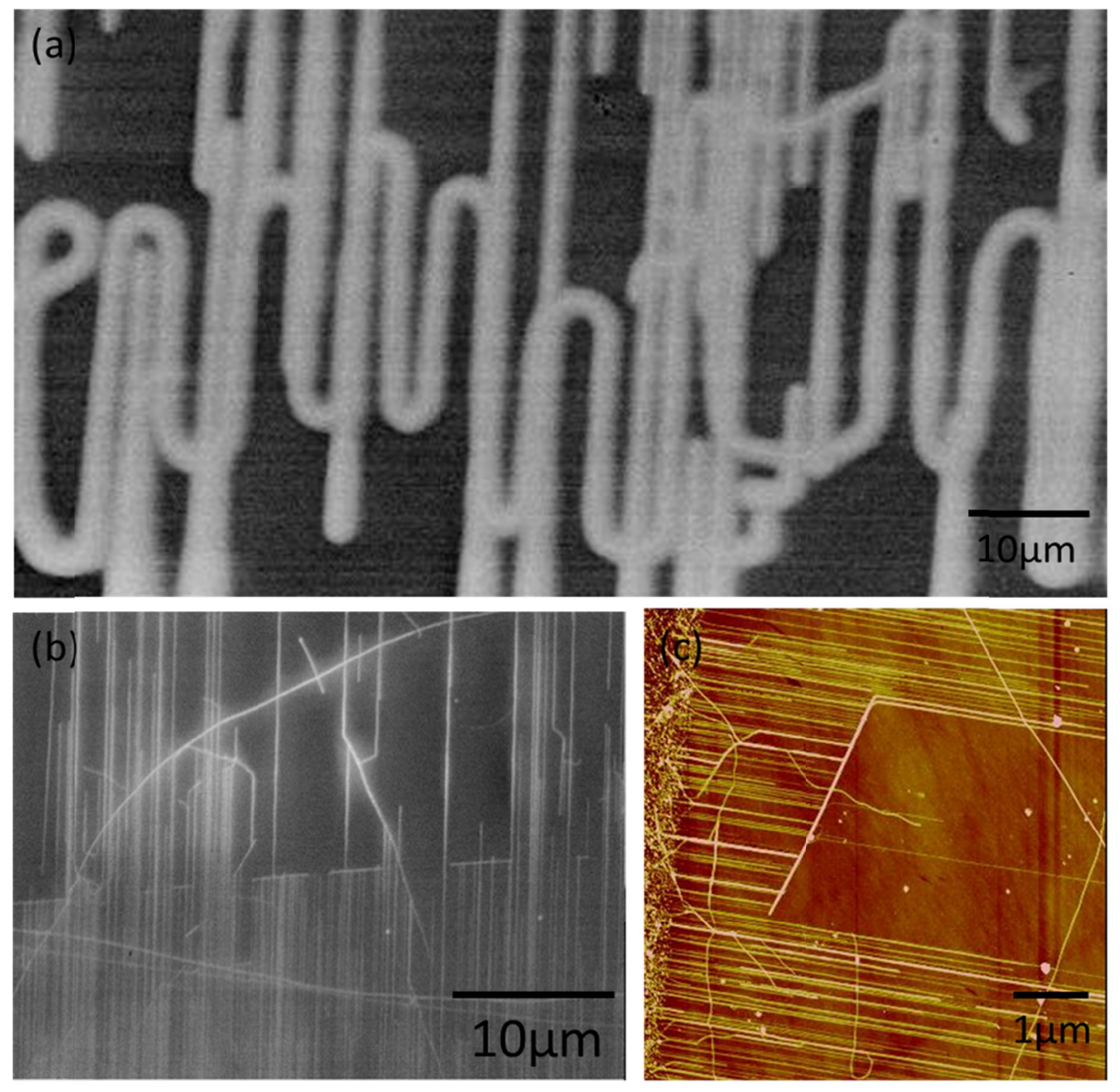

Figure 14: (a) Individual SWCNT turned 180 degrees for multiple times on the substrate. (b) SWCNT segments, grown in the random direction, functioned as nano-barriers that stopped the growth SWCNT grown along the [100] direction. (c) An AFM image of a nano-barrier.

The barriers function well as a result of the diameter of the lattice oriented SWCNTs being significantly smaller than the diameter of the nano-barrier CNTs. Even if the lattice oriented carbon nanotube goes beyond the nano-barrier, the growth can be easily terminated. Because the slits between the nano-barriers and quartz surface are the space through which the growth takes place, the growth can be terminated just beyond the barrier by strong frictions. However, a growth beyond the barrier is still possible if the diameter of the SWCNT is larger than the diameter of the nano-barrier. Desired 
lengths of SWCNTs can be achieved on a quartz surface by altering the locations of the catalyst and nano-barriers.

\subsubsection{Raman spectroscopy characterization of pristine samples of this research}

From the Raman spectra of SWCNTs, information can be obtained about the 1dementional phonon structure and electronic structures of the nanotubes, as well as information of their defects. Three characteristic bands are of special interest in Raman spectra of CNTs, the well-known radial breathing mode (RBM), D-band (disorder induced feature), and G-band (tangential phonon modes). Typically, the presence of RBM band is positioned in the range of $100-350 \mathrm{~cm}^{-1}$ and corresponds to the expansioncontraction of the nanotube in the radial direction. It is a signature for SWCNTs. For an as-grown sample, a large D-band peak compared with the G-band peak means a bad resonance condition and the G/D ratio is often used to indicate the structural quality of SWCNTs. By focusing on an individual SWCNT on a sparsely grown sample, the RBM and G-band can be used to select semiconducting or metallic SWCNTs [79].

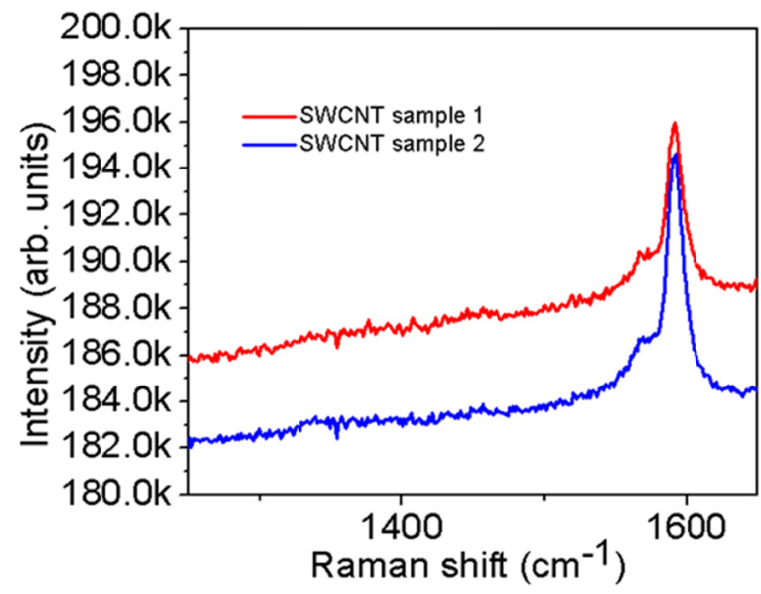

Figure 15: Raman spectra of two pristine samples give distinctive peaks at G-band but no obvious peaks at D-band. 
The as-grown samples (sample 1 and 2) have been characterized by Raman spectroscopy illuminated by an argon laser with an excitation wavelength of $514 \mathrm{~nm}$. No peak is observed at D-band (near $1340 \mathrm{~cm}^{-1}$ ) for either of the 2 samples. The distinctive relative intensity difference between the D-band $\left(\sim 1340 \mathrm{~cm}^{-1}\right)$ and G-band $\left(\sim 1580 \mathrm{~cm}^{-1}\right)$, as shown in Figure 15, infer that the defect density on the SWCNTs is low. This confirms the conclusion from Ding et al. [61] about the high quality of the sample. However, at the radial breathing mode range $\left(100 \sim 250 \mathrm{~cm}^{-1}\right)$, the single SWCNT sample overlapped with a strong peaks from the quartz substrate, no distinctive feature from SWCNTs can be identified in the spectra. Further calibration and sample transferring are needed for future studies.

\subsection{Conclusions}

Horizontally aligned SWCNTs were successfully synthesized on the thermally annealed St-cut quartz substrate. Pristine samples were characterized by SEM, AFM and Raman spectroscopy. The results indicated that these samples have good alignment $(88 \%$ alignment), small and uniform diameter $(1.4 \pm 0.3 \mathrm{~nm})$, and low defects. A conclusion could be arrived at as far as the reason responsible for the alignment of the nanotubes is concerned that the alignment was a result of the surface lattice structure of the selective cutting of the substrates. Annealing effects on the alignment and on the substrate surface were studied. The surface coverage of SWCNTs varied from one sample to another. The density of SWCNTs decayed exponentially in the first $30 \mu \mathrm{m}$ after they grew out of the edge of the catalyst band. However, the density became quite uniform thereafter as the result of catalyst particle migration, which was supported evidently by the phenomena such as serpentine SWCNT, and nanotube nano-barrier. A tip-growth mechanism of the 
SWCNTs was implied from the growth mechanism of the serpentine SWCNT and CNT nano-barrier function, which were discussed in this chapter.

Around the bending portion of a nanotube, the radial mechanical properties will be altered and the resistance can increase by more than an order of magnitude [23]. These samples with the intrinsic straightness are deformation-free and thus offer us a great chance to study the mechanical and electrical transport properties on perfectly structured SWCNTs. Moreover, the uniform SWCNTs coverage on the substrate in the vast area far from the catalyst region provides an ideal platform for nanotube ultrathin-film application such as SWCNT thin-film biosensors. 


\section{CHAPTER 4}

\section{Radial elasticity measurement of single-walled carbon nanotubes by atomic force microscopy}

\subsection{Background of this research}

The mechanical properties of carbon nanotubes have been studied for their promising applications in composite materials $[80,81]$ and in integrated circuit systems $[82,83]$. A single-walled carbon nanotube can be treated as a transversally isotropic material because of its tubular structure. Its elastic modulus and Poisson's ratio depend weakly on chirality when its radius $\left(\mathrm{R}_{\mathrm{cnt}}\right)$ is greater than $0.5 \mathrm{~nm}[18,84,85]$. By treating the AFM tip and silicon substrate as rigid, Barboza et al. [84] have investigated the effective radial modulus ( $\mathrm{E}_{\text {radial }}$ ) of SWCNT with diameters from 2 to $3 \mathrm{~nm}$, using contact mode AFM. However, because of the comparable values of the SWCNT's own deformation, DeBorde et al. [85] have cautioned against neglecting deformations of both the AFM tip and the silicon substrate. As the result of the contact force, contact mode AFM alone cannot directly measure D but can rather estimate its value, thus, requiring the further measurement of $\delta$ and $\mathrm{E}_{\text {radial. }}$.

The $E_{\text {radial }}$ has also been measured for multi-walled CNTs [21-23] and for SWCNTs [73] using nanoindentation technique. To ensure the applied vertical force to be equal to the normal force, the nanoindentation technique strictly requires an AFM tip to be positioned right above the ridge of the CNT. Moreover, the inevitable sample contamination from synthesis and post-growth manipulations gives rise to considerable differences in cross-sections and mechanical properties measured at different positions along a CNT [35]. It has also been pointed out that possible sample contamination 
resulted from synthesis and post-growth manipulation will affect cross-sections and mechanical properties of the CNTs along their length [23]. In this dissertation, a method is reported to measure the $\mathrm{E}_{\text {radial }}$ by combining well-calibrated tapping mode and contact mode AFM on the as-grown horizontally aligned SWCNTs with diameter smaller than 2 $\mathrm{nm}$. In order to minimize the random error of measurement of compression at individual positions on the CNT, in each measurement the compression of the sample under normal force was averaged over a certain length of the CNT. By employing Hertzian theory, this research specifically exploits the components of deformation of the AFM tip-SWCNTsubstrate system.

\subsection{Experimental design}

By using tapping mode AFM in air (Multimode AFM IIID, Veeco Metrology), the original diameter of the SWCNT is measured using an oscillating silicon cantilever of driving frequency $10 \%$ below its resonance and a small free-amplitude (12 nm). By adjusting the tapping mode set-point, the SWCNT can be compressed and recovered on the quartz substrate. It is found that the height reduction of the SWCNT is revisable up to $60 \%$, which agrees well with the reported result of $\sim 63 \%$ for SWCNT with $1.5 \mathrm{~nm}$ diameter on silica measured by a ac-mode AFM [85]. After the tapping mode test, contact mode is then used to scan the same SWCNT. A silicon tip on silicon nitrite cantilever (model-MSNL, Veeco) with calibrated spring constant $\mathrm{k}=0.010 \mathrm{nN} / \mathrm{nm}$ is employed for the contact mode imaging; the scanning direction (fast direction) is chosen to be perpendicular to the axis of the SWCNT. The typical radius of curvature $\left(\mathrm{R}_{\mathrm{tip}}\right)$ of the silicon tip used for this scanning process is evaluated to be $25 \mathrm{~nm}$ by using the formula [85], $\mathrm{R}_{\text {tip }}=\omega 2 / 8 \mathrm{~h}$, where $\omega$ and $\mathrm{h}$ are the apparent width and height of SWCNT in AFM 
image, respectively. The contact force between the scanning AFM tip and the SWCNT can be determined by Hooke's law, $\mathrm{F}_{\mathrm{z}}=-\mathrm{k} \cdot \Delta \mathrm{z}$, where $\Delta \mathrm{z}$ is the vertical cantilever deflection.



Figure 16: The schematic of the cross-section of AFM tip-SWCNT-substrate system when the AFM tip (with $R_{\text {tip }} \approx 20 R_{\text {cnt }}$ ) is going across the ridge of a SWCNT. It is assumed that the thickness of the native oxide layer on the $A F M$ is $\sim 2 R_{\text {cnt }}$.

Inevitably, native oxide layer (fused silica) will quickly form on silicon tip in air. It is expected the native oxide layer will have a thickness of $2 \sim 3 \mathrm{~nm}$ [35], which is equal or greater than $2 \mathrm{R} c n t\left(\mathrm{R}_{\mathrm{cnt}}\right.$ is the radius of the tested SWCNTs). Considering $\mathrm{R}_{\mathrm{tip}} \approx 25$ $\mathrm{nm} \geqq 20 \mathrm{R}_{\text {cnt }}$, the cross-section of tip-SWCNT-substrate system can be treated as a structure as shown schematically in Figure 16. Only the vertical displacement of the tipSWCNT-substrate system at the vertex of the SWCNT cross-section was measured, where the normal force on the SWCNT is in the $\mathrm{z}$-direction $\left(\mathrm{F}_{\mathrm{z}}=\mathrm{F}_{\text {normal }}\right)$. The lateral force 
has no contribution in the z-direction. The measured height $\mathrm{h}$, as shown in panels (d), (e) and (f) in Figure 17, is defined as the vertical distance between the two red arrows. The scanned segment of the SWCNT and the substrate are marked by two short green lines separated horizontally by $40 \mathrm{~nm}$ in all panel (a), (b) and (c). The measurement of $\mathrm{h}$ took place over a restricted portion on an individual SWCNT where apparent contaminations are avoided. The deformation of the AFM tip-SWCNT-quartz system $(\Delta \mathrm{h})$ in panel (e) (or panel (f)) under compression is calculated by subtracting the $\mathrm{h}$ value in panel (e) (or panel (f)) from the $\mathrm{h}$ value in panel (d) where the SWCNT is not compressed. The $\Delta \mathrm{h}$ is composed of three components. The first component of $\Delta \mathrm{h}$ is the indentation of quartzSWCNT system, which includes the indentation contribution from both the quartz and SWCNT. The second component of $\Delta \mathrm{h}$ is the indentation of the AFM tip by a rigid SWCNT (note, to avoid double accounting the SWCNT's compression, in the second component the SWCNT is treated as rigid rod). The first two indentation components will occur when the AFM tip is exerting normal forces directly on the top of the SWCNT, as indicated by the left red arrows in Figure 17(e) and 2(f). When the AFM tip scans the substrate, it also indents the substrate at the positions indicated by the right red arrows in Figure 17(e) and 2(f). The indentation between the AFM tip and the substrate is the third component of $\Delta \mathrm{h}$ and it will contribute negatively to the calculation of $\Delta \mathrm{h}$. Palaci et al. [8] predicted that the compression of a CNT between a rigid AFM tip and rigid substrate follows the first level approximation of Hertzian model with a contact area and an adhesion correction. Considering the three components of the indentation in this AFM tip-SWCNT-substrate system and the fact that the $\Delta \mathrm{z}$ measured by us includes the deflection caused by the adhesion, $\Delta \mathrm{h}$ can be written as: 
$\Delta \mathrm{h}=\left[\mathrm{F}_{\mathrm{z}} /\left(\mathrm{K}_{1} \sqrt{2 \mathrm{R}_{\mathrm{cnt}}}\right)\right]^{2 / 3}+\left[\mathrm{F}_{\mathrm{z}} /\left(\mathrm{K}_{2} \sqrt{\mathrm{R}_{*}}\right)\right]^{2 / 3}-\left[\mathrm{F}_{\mathrm{z}} /\left(\mathrm{K}_{3} \sqrt{\mathrm{R}_{\text {tip }}}\right)\right]^{2 / 3}$

Where $\mathrm{K}_{1}=(4 / 3)\left[\left(1-v_{\text {quartz }}{ }^{2}\right) / \mathrm{E}_{\text {quartz }}+\left(1-v_{\text {cnt }}{ }^{2}\right) / \mathrm{E}_{\text {radial }}\right]^{-1}, \mathrm{~K}_{2}=(4 / 3)\left[\left(1-v_{\text {tip }}{ }^{2}\right) / \mathrm{E}_{\text {tip }}\right]^{-1}$ and $\mathrm{K}_{3}=(4 / 3)\left[\left(1-v_{\text {quartz }}{ }^{2}\right) / \mathrm{E}_{\text {quartz }}+\left(1-v_{\text {tip }}{ }^{2}\right) / \mathrm{E}_{\text {tip }}\right]^{-1}$ are the reduced elastic moduli of the quartzSWCNT system, the "rigid SWCNT indenter"-AFM tip system, and the quartz-AFM tip system, respectively. $\mathrm{R} *=\left[1 /\left(2 \mathrm{R}_{\mathrm{cnt}}\right)+1 / \mathrm{R}_{\mathrm{tip}}\right]^{-1}$ is the reduced radius of curvature of the cylindrical SWCNT-spherical tip system [8]. The Young's modulus for a quartz substrate is $\mathrm{E}_{\text {quartz }}=76.5 \mathrm{GPa}$ [36], for $\mathrm{AFM}$ tip $\mathrm{E}_{\text {tip }}=74 \mathrm{GPa}$ (considering the native oxide layer), and the Poisson's ratio $v_{\text {tip }}=0.16, v_{\text {quartz }}=0.1815, v_{\text {cnt }} \approx v_{\text {graphene }}=0.17$ (when $\mathrm{R}_{\text {cnt }}>0.5 \mathrm{~nm}$ ) [86].

\subsection{Result and discussions}

In the ref. [8] a similar situation (contact between a cylinder and a sphere) has been discussed by Palaci et al. in 2005 using nanoindentation. The contact between a cylinder and a plane can be treated as the contact between a cylinder and a sphere with a very large radius. In this approximation, their definition of the reduced curvature $\left(1 / R^{*}\right)$ was used in considering the contact geometry, i.e., $R^{*}=\left[1 /\left(2 R_{\text {cnt }}\right)+1 / R_{\text {tip }}\right]^{-1}$ (it will be $\mathrm{R}^{*}=\left(1 / \mathrm{R}_{1}+1 / \mathrm{R}_{2}\right)^{-1}$, if $\mathrm{R}_{1}$ and $\mathrm{R}_{2}$ are the radii of two spheres $)$. The eqn. 2 for the contact of a cylinder and a sphere in ref. [8] $\quad\left(\mathrm{k}_{\mathrm{con}}=\beta\left[\mathrm{R} *\left(\mathrm{~F}+\mathrm{F}_{\mathrm{adh}}\right) / \mathrm{K}^{2}\right]^{1 / 3}\right.$, where $\mathrm{K}=(3 / 4)[(1-$ $\left.\left.\left.v_{\text {tip }}{ }^{2}\right) / \mathrm{E}_{\text {tip }}+\left(1-v_{\text {cnt }}{ }^{2}\right) / \mathrm{E}_{\text {radial }}\right]\right)$, is basically the first level approximation of Hertzian model, i.e., $\Delta h \propto \mathrm{F}_{\mathrm{z}}^{2 / 3}$, since the stiffness $\mathrm{k}=\mathrm{F}_{\mathrm{z}} / \Delta \mathrm{h}$. The model of contact between the cylinder and sphere will apply to a sphere indenting a cylinder (e. g., AFM tip on CNT) or a cylinder indenting a plane (e. g., CNT on the substrate). 




Figure 17: (a): High resolution tapping mode AFM image of the SWCNT. (b) and (c): contact mode images of the same region shown in panel (a), the white boxes show where the radial modulus measurement taking place for individual CNT. (d), (e) and (f): The averaged cross-sections of the boxed volumes in panel (a), (b) and (c), respectively. Height color bars are 0-4 $\mathrm{nm}$ in all three images. The contact forces are $\mathbf{0}, 7.4$, and $13.1 \mathrm{nN}$ for scanning image (a) (free-amplitude tapping), (b), and (c), respectively.

About the surface adhesion correction used by Palaci et al. $\left(\mathrm{F}_{\mathrm{adh}}\right)$ in the eqn. 2 in ref. [8], it can be seen from the force calibration plot (Figure 18) that in this case, the vertical cantilever deflection (e. g. $\Delta \mathrm{z}=25 \mathrm{~nm}$ in total) in the Hooke's law: $F_{z}=-k \cdot \Delta z$, is measured from the zero-force point to the set-point voltage (SPV) level (e.g., 1V). 
Therefore the $\Delta \mathrm{z}$ measured by us have already included the adhesion-caused deflection (from zero-force point to $0 \mathrm{~V}$ level).

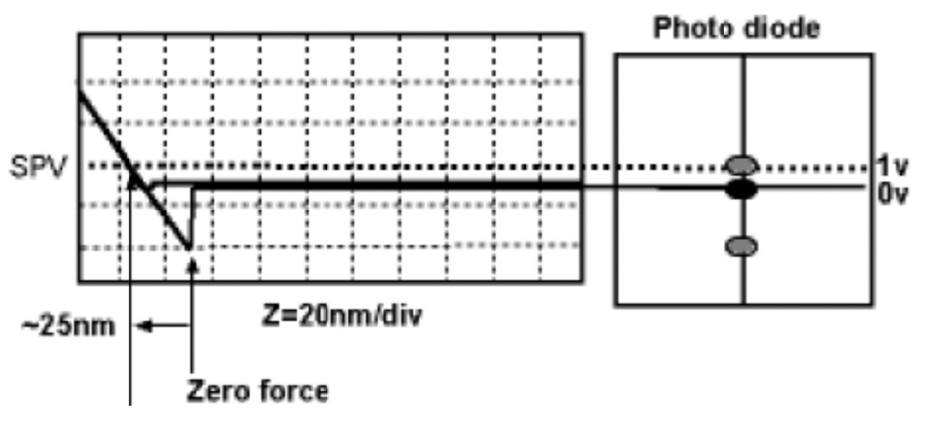

Figure 18: The force calibration curve of our AFM system.

The elliptic-shape contact-area coefficient $\beta$ added to the Hertzian model by Palaci et al. [8] is arguable in the case of this study, since the indentation of both our substrate and tip, which is comparable to the indentation value of SWCNT, can cause the contact-area shape to be a "shallow saddle". Therefore the $\beta$ was not included in the approximation of this study.

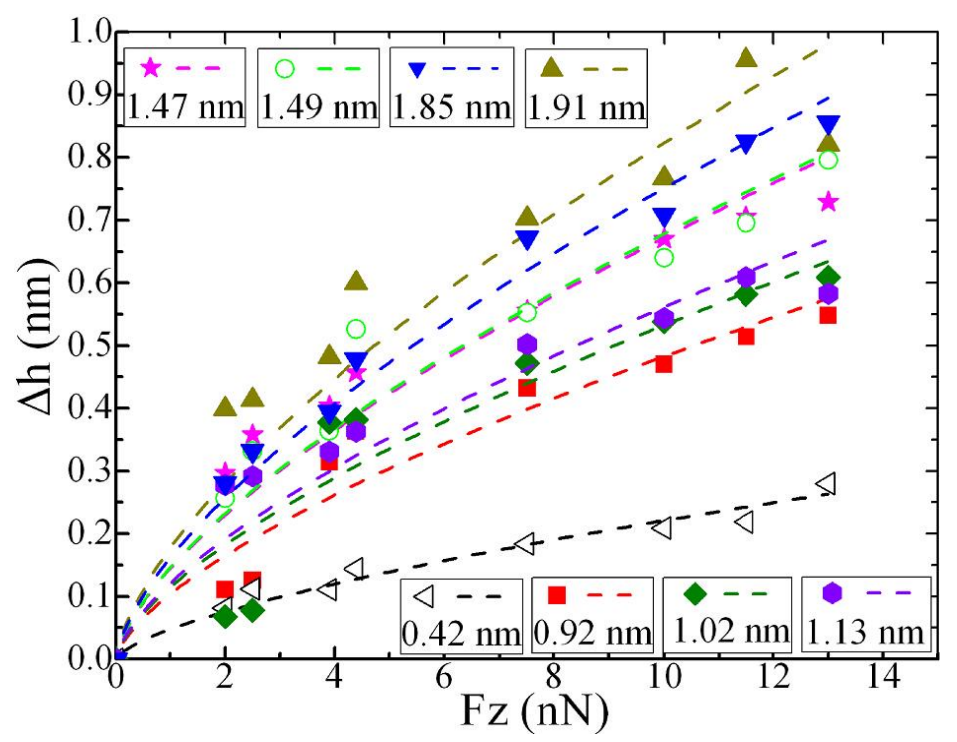

Figure 19: The measured $\Delta \mathrm{h}$ vs. Fz as well as the fitting to the experimental data by using the power function relation $\Delta h \propto F z^{2 / 3}$. From the top fitted curve to the bottom one, the diameter of SWCNTs decreases from 1.91 to $0.42 \mathrm{~nm}$. 
Figure 19 shows radial displacement $(\Delta \mathrm{h})$ vs. normal force $\left(\mathrm{F}_{\mathrm{z}}\right)$ data for eight individual SWCNTs scanned by free-amplitude tapping mode AFM and contact mode AFM with contact forces up to $13.1 \mathrm{nN}$. Up to $60 \%$ height compression is achieved with the largest applied contact force. The $\Delta \mathrm{h}$ vs. $\mathrm{F}_{\mathrm{z}}$ data are fitted with function $\Delta \mathrm{h}=\lambda \mathrm{F}_{\mathrm{z}}{ }^{2 / 3}$, where $\lambda$ is the fitting coefficient extracted from Eqn.1. The effective radial modulus was then calculated using the $\lambda$. The SWCNT with the smallest radius $(0.21 \mathrm{~nm})$ was excluded from the calculation for the fact that the approximation was valid for $\mathrm{R}_{\mathrm{cn}}>0.5 \mathrm{~nm}$. As the diameter of the nanotubes increases from 0.92 to $1.91 \mathrm{~nm}$, the measured $\mathrm{E}_{\text {radial }}$ decreases from 57 to $9 \mathrm{GPa}$. Figure 20 shows the comparison of the $\mathrm{E}_{\text {radial }}$ of SWCNTs with different radius from this experiment, the reported nanoindentation result [22], and the reported computational result [87]. The $\mathrm{E}_{\text {radial }}$ distribution with the tube radius for seven SWCNTs remarkably resembles the data pattern obtained by Chen et al. using the modified molecular structure mechanics (MSM) model [87]. In Chen et al.'s model, the force field between two bonded carbon atoms is modeled with continuum pseudorectangular beam elements. The pseudo-rectangular beam consists of a different bending rigidity along the two principal centroidal axes of the cross-section, the stiffness parameters of which are estimated based on the bond-angle variation energy and the weak inversion energy in molecular mechanics. It has been shown that the $\mathrm{E}_{\text {radial }}$ and radius $R_{\text {cnt }}$ of SWCNTs follow the relationship $E_{\text {radial }} \propto R_{\text {cnt }}^{-\alpha}$, where $\alpha$ equals to 2 for SWCNT in pure "hard phase" and $\alpha$ equals to 3 for SWCNT in pure "soft phase" [22, 88]. The experimental data from our research and Chen et al.'s computational data reported in ref. [87] were fitted and it was found that the $\alpha$ values are 2.812 and 2.695 for my data and Chen et al.'s data, respectively. It is expected that both of the $\alpha$ values from 
Chen et al.'s and from this study will fall between 2 and 3 because both the results have averaged the effect of the transition of SWCNT from a hard phase to a soft phase on the $E_{\text {radial }}$. However the nanoindentation data reported in ref. [22] is out of this range $(\alpha=0.818)$, as shown in Figure 20.



Figure 20: Ther data of measured $E_{\text {radial }} v s$. $R_{\text {cnt }}$ (black squares) compares with nanoindentation experimental data (blue dots, see [8]) and modified MSM calculation data (red triangles, data courtesy of Mr. Y.L. Liu).

The measurements in this dissertation was compared with the previous experimental results obtained by Barboza et al. [84], who has developed a model from the result of first-principles calculation. Unlike Chen et al.'s model, Barboza et al.'s model treats $\mathrm{E}_{\text {radial }}$ as a function of nanotube strain instead of a constant for a given SWCNT. The measurements of large diameter SWCNTs (diameter close to $2 \mathrm{~nm}$ ) in our study are in agreement with Barboza's data and modeling [84]. However, measurements of 
SWCNTs with smaller diameters deviate from Barboza et al.'s model. It is argued below that this can be explained by the compressibility of the substrate and the AFM tip. Figure 21 is the renormalized data in the plot of $\mathrm{Fd}^{3 / 2}\left(2 \mathrm{R}_{\text {tip }}\right)^{-1 / 2}$ vs. Radial strain, where $\mathrm{d}$ is from 0.42 to $1.91 \mathrm{~nm}$, and $\mathrm{R}_{\text {tip }}$ is $25 \mathrm{~nm}$. The same theoretical curve of Eqn. 1 with the same scale as that in the paper of Barboza et al. (with $\alpha=1.2 \times 10-18 \mathrm{~J}$ ) was reproduced below using the software Origin 8.

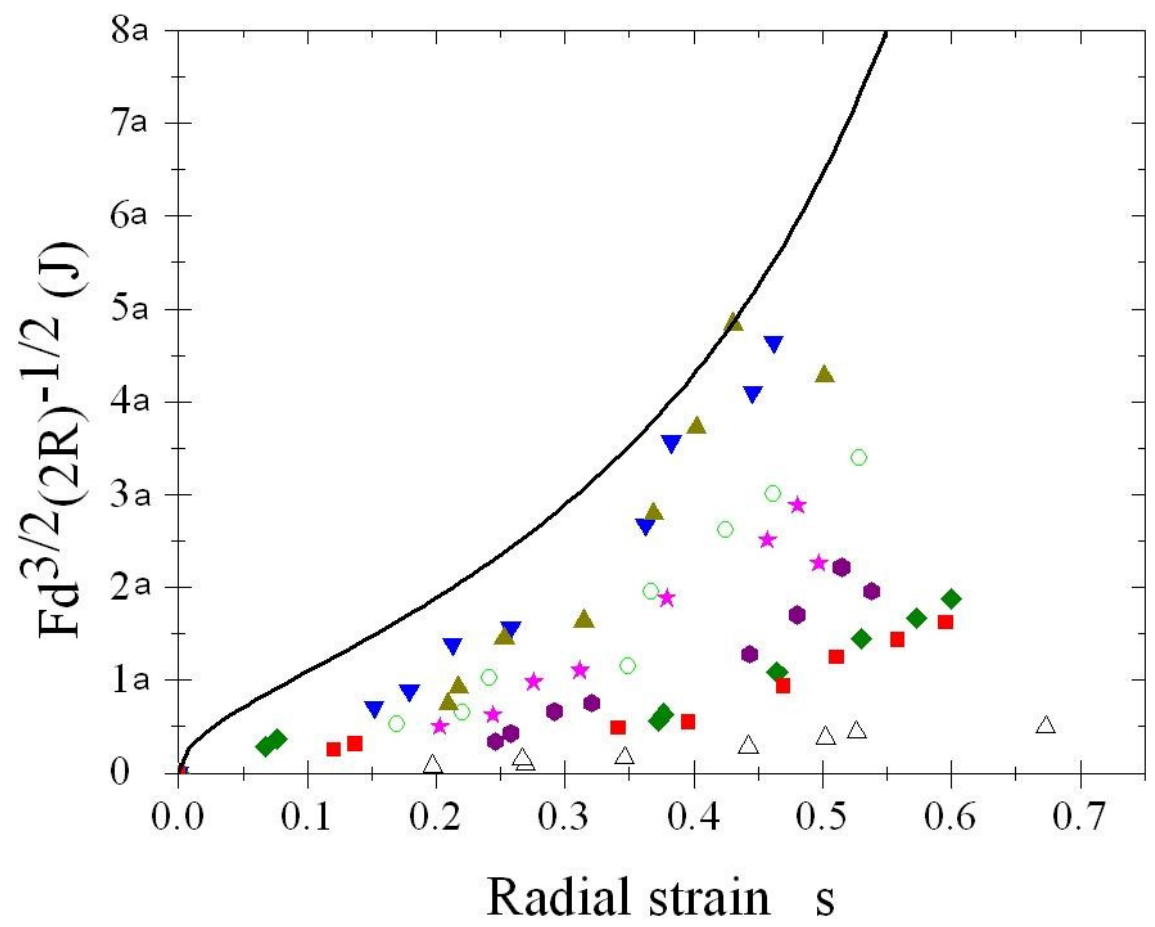

Figure 21: The renormalized data in the plot of $F^{3 / 2}\left(2 R_{\text {tip }}\right)^{-1 / 2} v s$. Radial strain.

Compare the data from Barboza et al., my data points for SWCNTS with smaller diameters (less than $2 \mathrm{~nm}$ ) scatters below the curve. It can be observed that the smaller the diameter of SWCNT is (same symbols of the data points used in Figure 19), the further apart the data points are from the theoretical curve. It is expected that this disagreement is mainly resulted from the large indentation of the substrate and tip by 
SWCNTs with small diameter. Therefore, the indentation of quartz substrate and AFM tip by SWCNTs with different diameters was discussed theoretically in Figure 23.

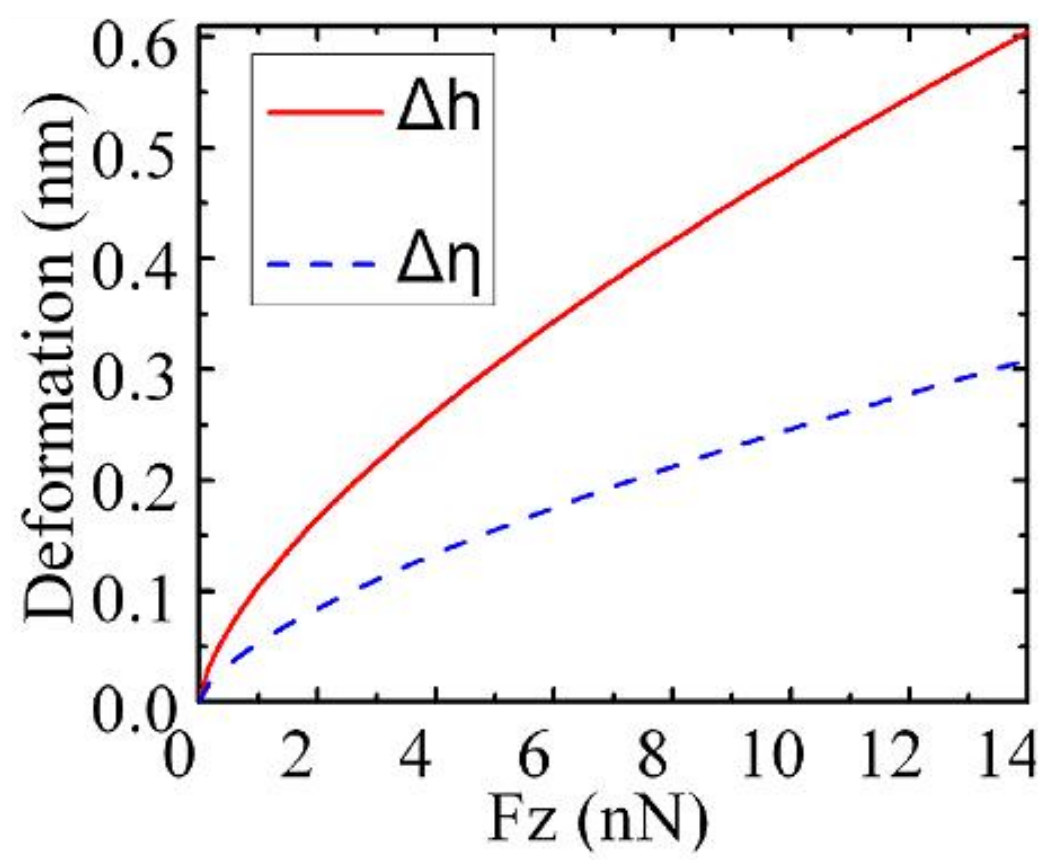

Figure 22: $\Delta \mathrm{h}$ and $\Delta \eta$ as different functions of Fz for a SWCNT with $0.92 \mathrm{~nm}$ diameter.

The last two terms in Eqn.1 vanish if the substrate and AFM tip are treated as perfectly rigid materials. In this ideal case, the deformation is only on the SWCNT. The deformation of the SWCNT, $\Delta \eta$, can be described using $\Delta \eta=\left[\mathrm{F}_{z} /\left(\mathrm{K}_{1} \sqrt{2 \mathrm{R}_{\mathrm{ctt}}}\right)\right]^{2 / 3}$, where $\mathrm{K}_{1}=(4 / 3)\left[\left(1-v_{\text {cnt }}{ }^{2}\right) / \mathrm{E}_{\text {radial }}\right]^{-1}$. Therefore, the $\Delta \eta$ can be calculated theoretically with the measured values of $E_{\text {radial. }}$. Figure 22 shows $\Delta h$ and $\Delta \eta$ as a function of $F_{z}$ for the SWCNT with $0.92 \mathrm{~nm}$ diameter. Note, both $\Delta \mathrm{h}$ and $\Delta \eta$ are proportional to $\mathrm{F}_{\mathrm{z}}^{2 / 3}$. Therefore, $(\Delta \mathrm{h}$ $\Delta \eta) / \Delta \eta$ reflects the ratio of the deformation contributions from the AFM tip and substrate to the deformation contribution from the SWCNT under the same $F_{z}$. The deformation $(\Delta \eta)$ of SWCNT between rigid quartz substrate and rigid AFM tip was calculated theoretically, and compared with the deformation $(\Delta \mathrm{h})$ of the tip-SWCNT-quartz system under the same normal force $\left(\mathrm{F}_{\mathrm{z}}\right)$. Figure 23 shows $(\Delta \mathrm{h}-\Delta \eta) / \Delta \eta$ as a function of the 
SWCNT diameter. As the diameter of the SWCNT increases from 0.92 to $1.85 \mathrm{~nm},(\Delta \mathrm{h}-$ $\Delta \eta) / \Delta \eta$ decreases from 0.96 to 0.15 and becomes flat. The data pattern in Figure 23 indicates that a SWCNT with small diameter will cause more significant indentation on the substrate and tip than a SWCNT with big diameter. This is attributed to the large $E_{\text {radial }}$ of the SWCNT with small diameter and its small contact area with the substrate and tip.



Figure 23: The data of $(\Delta h-\Delta \eta) / \Delta \eta$ vs. diameter for 7 different SWCNTs.

In addition to the substrate and tip indentation, it is worth noting that $\mathrm{R}_{\text {tip }}$ has a major impact factor on this renomalization even though it plays a minor role in the calculation in this dissertation (since in our case, $R_{\text {tip }}>>R_{\text {cnt }}$ in the Eqn. 4.1). It is known that different AFM tips are used by Barboza et al. (claimed in the Fig. 4.1 caption of ref. [84] ), however the data for the compression in our study was collected by one contact mode AFM tip. 


\subsection{Conclusion}

In conclusion, it has been demonstrated here an efficient method of measuring the effective radial modulus ( $\left.\mathrm{E}_{\text {radial }}\right)$ of SWCNT by applying well-calibrated tapping mode and contact mode AFM upon horizontally aligned SWCNT samples. The measured $\mathrm{E}_{\text {radial }}$ decreases from 57 to $9 \mathrm{GPa}$ with the increase of the SWCNT diameter from 0.92 to 1.91 $\mathrm{nm}$. The experimental result is consistent with the computational data obtained using the modified MSM model. However, the data give different analytical result from that reported by Barboza et al. mainly because of the deformation of substrate and AFM tip by small diameter SWCNT. Further calculation with the measured $E_{\text {radial }}$ indicates that under the same normal force $\left(\mathrm{F}_{\mathrm{z}}\right)$ the deformation of this quartz-SWCNT-silicon tip system $(\Delta \mathrm{h})$ can be as much as $96 \%$ more than the deformation of SWCNT compressed between rigid substrate and AFM tip $(\Delta \eta)$. 


\section{CHAPTER 5}

\section{Electrical/electrochemical properties and the biosensing of single-walled carbon}

\section{nantoubes}

\subsection{Background of this research}

Various carbon materials have been used extensively as electrodes in analytical electrochemistry as the result of several important attributes including easy access, chemical and mechanical stability, wide electrochemical potential window in aqueous and organic solution, and biocompatibility [32]. Carbon nanotubes (CNTs) have outstanding structural and electronic properties compared with conventional carbon materials used in electrochemistry such as glassy carbon (GC), graphite, and carbon fiber [32, 33]. The advantages of using CNT as electrodes for electrochemistry includes: (1) CNTs have molecular size diameters and high aspect ratio. (2) CNTs are mechanically robust and thermally, electrically and electrochemically stable both in air and in electrolyte. (3) CNTs have high electrical conductivity and low capacitance. (4) CNT electrodes often show faster electron transfer kinetics than traditional carbon electrodes. (5) CNTs show electrocatalytic activity and can reduce overpotential.

In the last decade, numerous electrochemical sensing mechanisms and devices built with CNT have been reported [33]. Our fundamental understanding of the electrochemical properties of CNT devices has been significantly improved by the studies on individual single-walled carbon nanotubes (SWCNTs) [89]. Because of the challenges of sorting different type of CNTs in large scale, applications on the basis of large quantity of CNTs are more feasible in the short them. Large quantity of CNTs, in different forms, have been studied for various practical electrochemical sensing applications [90]. 
Especially, CNT thin film attracted most attentions. Numerous methods have been developed to prepare CNT thin films and can be broadly classified into two categories, solution-based deposition and direct growth [91]. Typical examples are membrane filtration [92] and chemical vapor deposition (CVD). The use of CVD method enables the direct growth of pristine CNT thin film on insulating surfaces, without the need for further purification, owning to the reduced amorphous carbon and metal content. Recent studies have shown that SWCNTs, arranged in a planar, extremely low surface coverage $(<1 \%)$ random network, on an insulating substrate, are extremely promising electrodes for low concentration electrochemical detection $[93,94]$ In addition to random network, horizontally aligned SWCNTs arrays have also been successfully prepared by CVD method. The alignment of CNT arrays can play important roles in the electrical properties of CNT thin film based FET devices [38, 91, 95]. Very recently, enhanced biosensing performance of FET device based on aligned CNT arrays has been reported [96]. The enhancement is attributed to the significantly reduced number of CNT-CNT junctions. However, the electrochemical properties of horizontally aligned SWCNT arrays have not been systematically investigated.

In this dissertation, facile method was developed to prepare high performance electrochemical biosensors based on horizontally partially aligned CNT ultrathin films. The CNT growth is self-guided on a single-crystalline substrate, ST-cut quartz substrate. The as-grown SWCNT arrays have high degree of alignment ( $>88 \%$ alignment). The high degree of alignment significantly reduced the number of CNT-CNT junctions existing in random CNT networks. Meanwhile, the small amount of misaligned CNTs seems beneficial for increasing the number of conductance pathways at long distance 
through percolation transport. A sub-monolayer of SWCNTs was therefore obtained with very low surface coverage $(<1 \%)$. Importantly, such thin film uniformly covered the whole substrate, which leads to greatly simplified fabrication process. It was easy to achieve micro- or nanoelectrode electrochemical performances using $\mathrm{mm}$ sized substrates. The fabricated electrochemical devices showed relatively high electrical conductivity, small capacitive current, low detection limit, fast response time, high stability and reproducibility. We investigated cyclic voltammograms of several wellstudied redox molecules and proteins on the CNT thin film electrode surfaces, which improved our understanding of the electrochemical performance of these CNT thin films and the interactions between these molecules and the CNT surface. This device has also demonstrated the good sensitivity, fast response, high stability and anti-fouling capability for electrochemical biosensing using neurotransmitter dopamine as an example. In these experiments, there is no surface treatment for the CNTs. Better sensitivity and selectivity are expected after adopting well-developed CNT chemical modification techniques. Along with the advantage of transparent substrate, this simple device will provide an ideal platform for further developing CNT based electrochemical biosensing, especially for the sensing of live cell activities when combining with optical microscopes.

\subsection{Experimental design}

\subsubsection{Materials, Chemical reagents and solution preparation}

All the chemical reagents are purchased from Sigma Aldrich and Arcos Organics and used without further purification. All solutions are prepared using deionized (DI) water $(\sim 18 \mathrm{M} \Omega)$ from water purification system (Ultra Purelab system, ELGA/Siemens). 
The prepared $\mathrm{KCl}$ and $\mathrm{PBS}$ solution is filtered through a $0.2 \mu \mathrm{m}$ filter. Before use, pure Argon is bubbled through the solution for 20-30 minutes to reduce oxygen concentration.

\subsubsection{CNT growth and device fabrication}

$0.5 \mathrm{~mm}$ thick ST-cut single-crystal quartz substrate (Hoffman Materials, Inc., single side polished; MTI Corp. double side polished) was annealed at $950{ }^{\circ} \mathrm{C}$ in nitrogen for $\sim 8 \mathrm{~h}$ prior to the CNT growth. A strip of cobalt catalyst (about $2.5 \mathrm{~mm}$ in width and $0.2 \mathrm{~nm}$ in thickness) was patterned using a shadow mask and deposited by an electronbeam evaporator (EGN4, Oxford Applied Research) onto the substrate. The strip was oriented perpendicular to the $\mathrm{X}$ axis of the quartz crystal, corresponding to the growth direction of the aligned SWCNTs. CNTs were grown by CVD method using ethanol as carbon source at $925^{\circ} \mathrm{C}$ in $50 \mathrm{sccm} \mathrm{Ar}$ and $50 \mathrm{sccm} \mathrm{H}_{2}$ flow for $12-15 \mathrm{~min}$. The main characteristics of the CNT thin film under the same growth condition are quite reproducible.

After CNT growth, an e-beam deposited gold electrode (50nm thick) was patterned at the catalyst region using a shadow mask. A $100 \mathrm{~nm}$ thick $\mathrm{SiO}_{2}$ layer was deposited by the same e-beam evaporator to partially cover the gold electrode and CNTs area. The $\mathrm{SiO}_{2}$ layer was served as a barrier layer to eliminate electrolyte leakage paths between gold electrode and exposed CNT area during electrochemical measurement. The top view of a device is shown in Figure 24 (b).

A liquid cell was made by polydimethylsiloxane (PDMS) with a punched hole ( $\sim 2.2 \mathrm{~mm}$ in diameter and $\sim 12 \mu \mathrm{L}$ in volume) in the middle, which is used to define the exposing area of CNT thin film to electrolyte. During the measurement, the liquid cell is 
sealed tightly to the chip surface. For flowing sensing measurement, the top of the liquid cell is closed and the solution can be exchanged through the inlet and outlet of the liquid cell using a syringe pump.

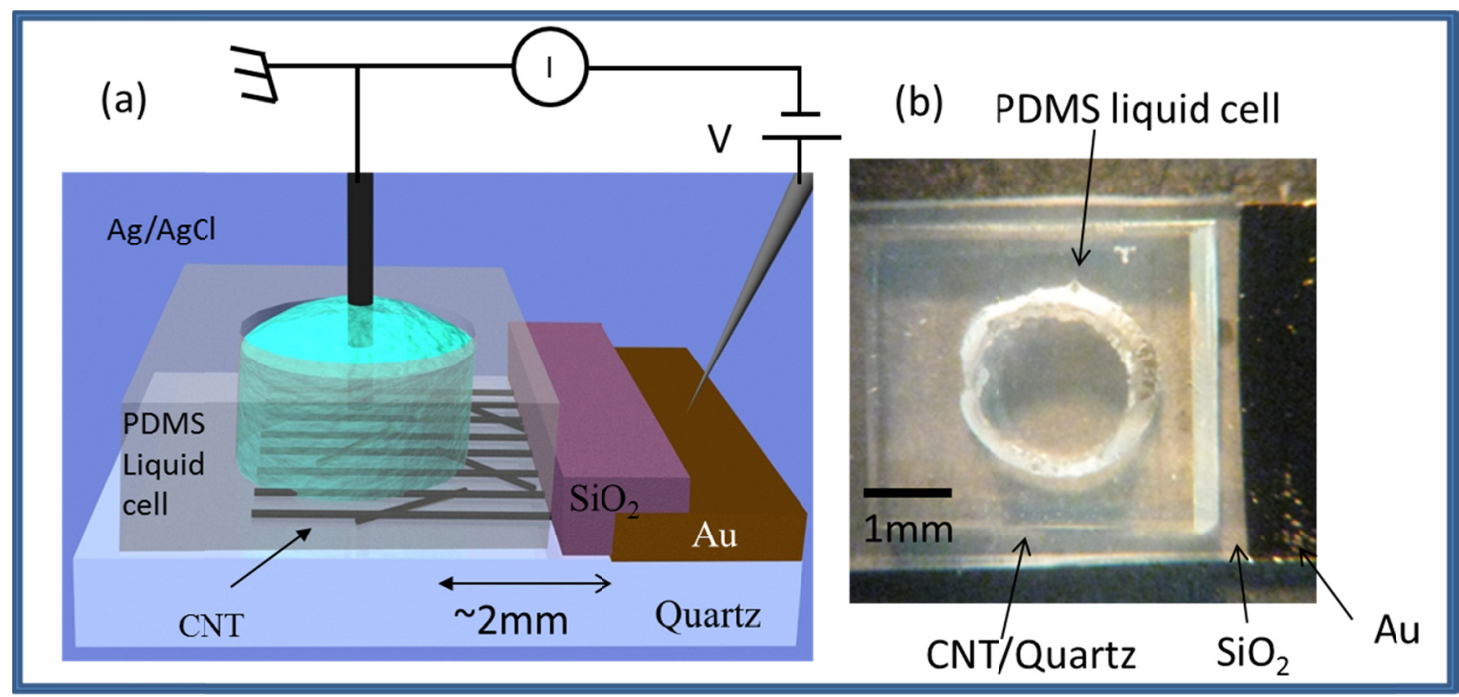

Figure 24: (a) Scheme of the device and electrochemical measurement connections. (b) The top view of the fabricated device.

\subsubsection{Measurement}

The electrochemical experiment setup and connections are illustrated in Fig ure 24(a). Because the electrochemical current is low $(\sim n A)$, the potential drop $i R_{s}\left(R_{s}\right.$ is the solution resistance) is very small and the electrode polarization is negligible. Therefore, counter electrode is not used in these measurements. A probe station is used to make electrical connection to the device. A quasi $\mathrm{Ag} / \mathrm{AgCl}$ electrode is used as the reference electrode, which is prepared by dipping a clean $0.25 \mathrm{~mm}$ diameter $\mathrm{Ag}$ wire into bleach for 30 minutes. The quasi $\mathrm{Ag} / \mathrm{AgCl}$ electrode was calibrated against a standard $\mathrm{Ag} / \mathrm{AgCl}$ electrode (MF-2078, BAS, 2M KCl) using gold reduction peak in acid and a shift of 70 $\mathrm{mV}$ was observed (i.e., a $0.3 \mathrm{~V}$ potential vs. quasi $\mathrm{Ag} / \mathrm{AgCl}$ electrode equals $0.37 \mathrm{~V}$ 
potential vs. standard $\mathrm{Ag} / \mathrm{AgCl}$ electrode). The cyclic voltammetry, I-V and I-t curves are collected with a Keithley Source Measure Unit (2636A, Keithley Instruments, Cleveland, Ohio). The measurement setup is placed in a home-built Faraday cage to reduce noise. For flow sensing experiments, a syringe pump (NE-1000, New Era Pump System Inc.) is used to control the flow rate of solution being injected. All the measurements are performed at room temperature.

\subsection{Results and discussions}

\subsubsection{Device characterization by electrical transport tests}

On the basis of the area density and CNT average diameter, which are characterized in chapter 3, The exposed CNT thin film surface area in the liquid cell were estimated to be about $3.8 \times 10^{-5} \mathrm{~cm}^{2}-2.5 \times 10^{-4} \mathrm{~cm}^{2}$. Raman spectra (see Fig. 3.9) of the pristine CNTs are also collected. No peak is observed at D-band (near $1340 \mathrm{~cm}^{-1}$ ), suggesting low level of defects for these partially aligned CNT thin films. 4 two-terminal devices were also fabricated and the electrical conductivity of the CNT thin film was characterized. A current-voltage (IV) curve of a typical device with electrode width (w) $5 \mathrm{~mm}$ and electrode separation length $(l) 4.5 \mathrm{~mm}$ is shown in Figure 25(a). The IV curve is a straight line and a sheet resistance $\left(R_{S}\right)$ of $1.13 \mathrm{M} \Omega$ /square is derived from the slope of the IV curve ( $1 / R)$ using equation $R=R_{S}(l / w)$. Contact resistance is ignored here, which is normally 2 orders smaller [97]. The derived $R_{s}$ values are between 1 and $4 M \Omega /$ square for all the measured devices. This sheet resistance is much bigger than the typical values of indium titanium oxide (ITO) glass or thick CNT thin film. This is because only a few CNTs in the film can bridge two electrodes separating a distance of $4.5 \mathrm{~mm}$. The misaligned CNTs play important roles in introducing new transport pathways between 
two electrodes. Therefore, the electrical transport is dominated by percolation transport and the carrier mobility is limited by the CNT-CNT junction resistance [38].

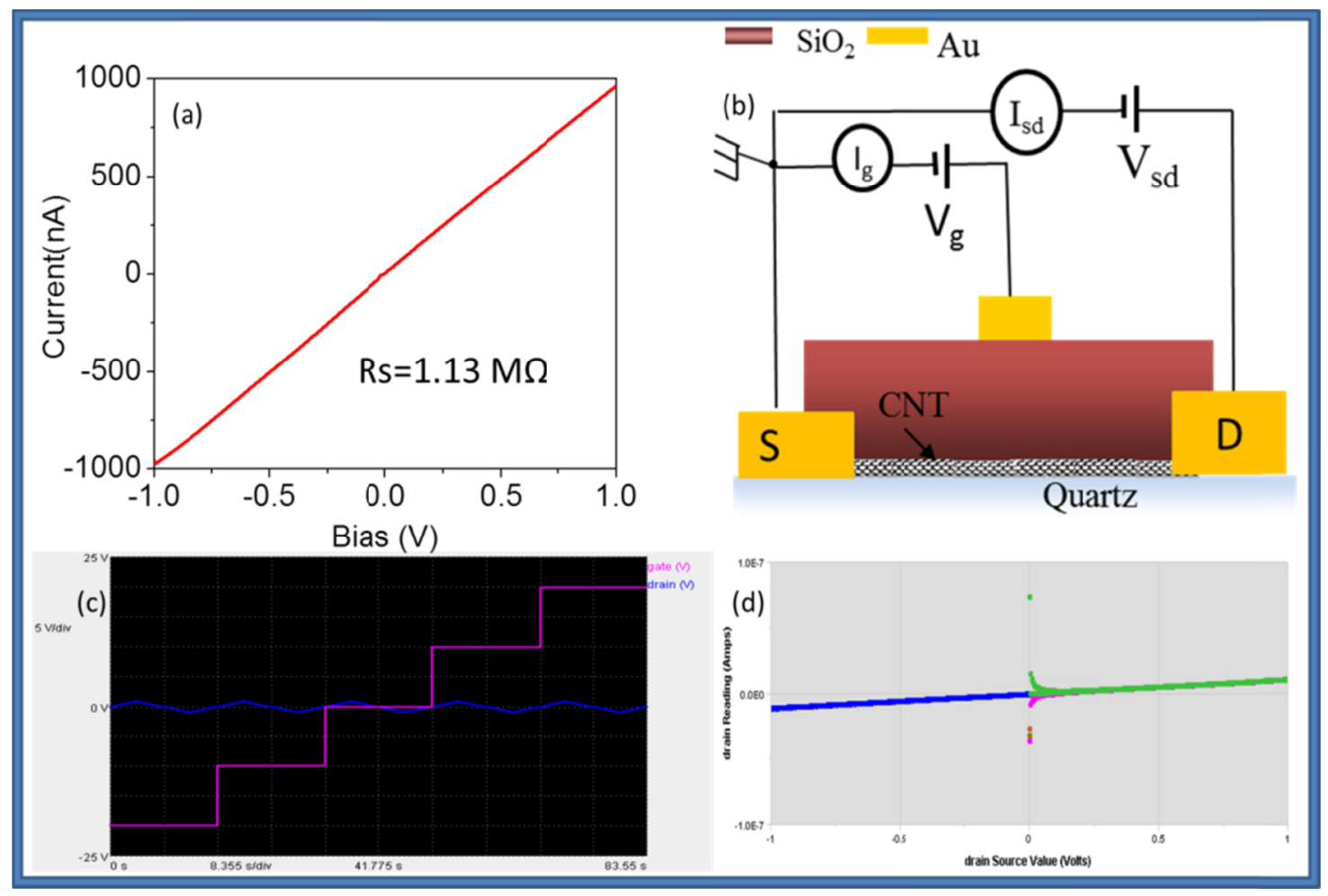

Figure 25: (a) A typical IV characteristics of a 2-terminal sample. (b) The scheme of the fabricated MOS-FEM based on my sample. (c) The applied $V_{\text {sd }}$ (blue) and $V_{g}$ (red). (d) 5 source-drain IV curves, indicated by 5 colors.

To further confirm the transport mechanism, the electronic properties of the CNT thin film were studied with $4.5 \mathrm{~mm}$ channel length by fabricating MOS-FET (Figure 25 (b)). A sweeping window from $-1 \mathrm{~V}$ to $1 \mathrm{~V}$ was applied between source and drain $\left(\mathrm{V}_{\mathrm{sd}}\right)$ and 5 different gate biases $\left(\mathrm{V}_{\mathrm{g}}\right.$, stepped from $-20 \mathrm{~V}$ to $\left.20 \mathrm{~V}\right)$ were applied between source and gate (Figure $25(\mathrm{c})$ ). However, 5 linear IV curves, corresponding to 5 different $\mathrm{V}_{\mathrm{g}}$, were obtained between source and drain, which overlapped with each other. No gating effect was observed in the applied $\mathrm{V}_{\mathrm{g}}$ range (Figure $25(\mathrm{~d})$ ). This is different from the $\mathrm{p}$ type FET performance of devices based on aligned SWCNT arrays with a much shorter 
channel length [16]. This result confirmed that the transport is dominated by the conduction through CNT-CNT junctions for the CNT thin film with millimeter length. This length scale is not suitable for FET device, but is desirable to be used as a working electrode for electrochemical measurements. Because the acquired electrochemical (EC) currents in my experiments are normally in the range of $\mathrm{nA}$, the high sheet resistance will not affect the EC current.

It is also interesting to note that the device is transparent. The optical transparency of the CNT thin film covered quartz substrate is measured by UV-vis spectroscopy at visible light range $(400-800 \mathrm{~nm})$ and the transmittance is about $97-99 \%$ at light wavelength $550 \mathrm{~nm}$. This transmittance is slightly better than ITO glass.

\subsubsection{Electron transfer kinetics}

For a newly developed working electrode, it is always important to characterize the heterogeneous electron transfer (HET) at the electrode. It provides a means to measure electron transfer kinetics and interactions between molecules and CNT surfaces. Considering the difference between redox systems [32], HET rate constants of a series of redox systems were studied at the aligned SWCNT thin film. These molecules also interact differently with the CNT surface, leading to different standard electron transfer rate. 30 devices have been fabricated and measured and 27 devices (90\%) yielded quite reproducible results that will be discussed below. The remaining 3 devices gave no or poor results, which may be attributed to the leakage or breakdown of devices.

The study started with a well-studied redox molecule, potassium ferrocyanide $\left(\mathrm{K}_{4} \mathrm{Fe}(\mathrm{CN})_{6}\right)$. As shown in Figure 26 (a), the cyclic voltammogram $(\mathrm{CV})$ of $1 \mathrm{M} \mathrm{KCl}$ solution was first obtained at $200 \mathrm{mV} / \mathrm{s}$ scan rate. In the potential window, no faradic 
current was observed and the interfacial capacitance was about $8.5 \mathrm{nF}$, which was very small, comparing with conventional thin film electrodes. Small capacitance will lead to a higher detection limit and fast response time. When $25 \mu \mathrm{m} \mathrm{K}_{4} \mathrm{Fe}(\mathrm{CN})_{6}$ was added into the solution, oxidative current appeared at positive potential as shown in Fig. 3A. The slightly increased current at negative potential is attributed to reduction current, which is much smaller than the oxidative current. The CV shape is very stable in consecutive sweeps. However, No diffusion-limited plateau was observed in the potential window.

The voltammetric curve shape is controlled by thermodynamics, mass transport and the molecule-CNT surface interactions. The missing of diffusion limited plateau and the much smaller reduction current within the scanned potential window suggests the concentration of reduced species is much bigger than the oxidized species near CNT electrodes even at the very positive potential. This may be the result of the following reasons. First, the mass transport of redox molecule to CNT electrode is very effective. Because of the small critical dimension of individual SWCNT and the sub-monolayer geometry of CNT thin film, mass transport can be highly effective. Second, the charge transfer process is slowed. The slowly rising slope and stretched shape of the CV indicated a smaller charge transfer rate. The fitting to a Bulter-Volmer model in the next paragraph confirmed the charge transfer rate is lower than the reported results for individual SWCNT. Therefore, the concentration of oxidized species is always low near the CNT thin film surface even at a more positive potential. Reduced species will continue to be oxidized and the CV shape will deviate from the classic nernstian curve shape. Similar phenomena have been reported for some individual SWCNT devices [89]. 
The steady-state voltammetric responses of the nanotube nanoprobes can be described by using a modified Butler-Volmer model which combines the heterogeneous electrode kinetics with the diffusive mass transport. In Butler-Volmer model, the diffusion-limited current, $i_{\mathrm{d}}$, is the limiting value (plateau) of a faradaic current that is approached as the rate of charge-transfer to an electrode is increased. The $i_{\mathrm{d}}$ can be approached by increasing the electric potential or decreasing the rate of mass transfer to the electrode. Eqn. 5.1 describes the faradic current, $i$, at an working electrode in terms of the overpotential, $E-E_{0}^{\prime}$.

For our device, the electrode kinetics is mainly contributed by the side walls of the CNTs. The oxidative and reductive current for a one-step one-electron process can be written as:

$i=\frac{i_{d}}{1+e^{\mp F\left(E-E_{0}^{\prime}\right) / R T}+K_{0}^{-1} e^{\mp F \beta\left(E-E_{0}^{\prime}\right) / R T}}$

where $i_{\mathrm{d}}$ is the diffusion-limit current, $R$ is the molar gas constant, $T$ is the absolute temperature, $F$ is the Faraday constant, $E$ is the applied potential, $E_{0}^{\prime}$ is the formal potential of the redox couple, and $K_{0}$ is the dimensionless heterogeneous rate constant. $K_{0}=F A C k^{0} / i_{\mathrm{d}}$, where $A$ is the surface area of the CNT thin film (estimated from SEM and AFM images), $C$ is the bulk concentration of the redox molecule and $k^{0}$, is the standard heterogeneous rate constant. In eqn. 5.1, the negative sign and $\beta=1-\alpha$ are used for oxidative current, and the positive sign and $\beta=\alpha$ are used for reductive current, where $\alpha$ is the transfer coefficient. Eqn. 5.1 (negative sign) has been used to fit the oxidative current of $\mathrm{K}_{4} \mathrm{Fe}(\mathrm{CN})_{6}$ in Figure 26 (a). The small reduction current is ignored here and assuming the concentration of $\mathrm{Fe}(\mathrm{CN})_{6}{ }^{4-}$ is the same as in the bulk. The standard rate constant $k^{0}$ is 
$0.14 \mathrm{~cm} / \mathrm{s}$. The standard rate constant normally obtained is between $0.03-0.2 \mathrm{~cm} / \mathrm{s}$, the variation mainly originated from the variation of CNT surface area. The obtained charge transfer rate is about 1-2 orders smaller than the results from individual SWCNTs (side walls) $[89,98]$. The decrease can be attributed to reduced carrier mobility through CNTCNT junctions and possible contaminations in the thin film. The reductive current of anion $\mathrm{Fe}(\mathrm{CN})_{6}{ }^{3-}$ were also measured and fitted (see Figure 26 (c)). The charge transfer rate is normally very close between $\mathrm{Fe}(\mathrm{CN})_{6}{ }^{4-}$ and $\mathrm{Fe}(\mathrm{CN})_{6}{ }^{3-}$.
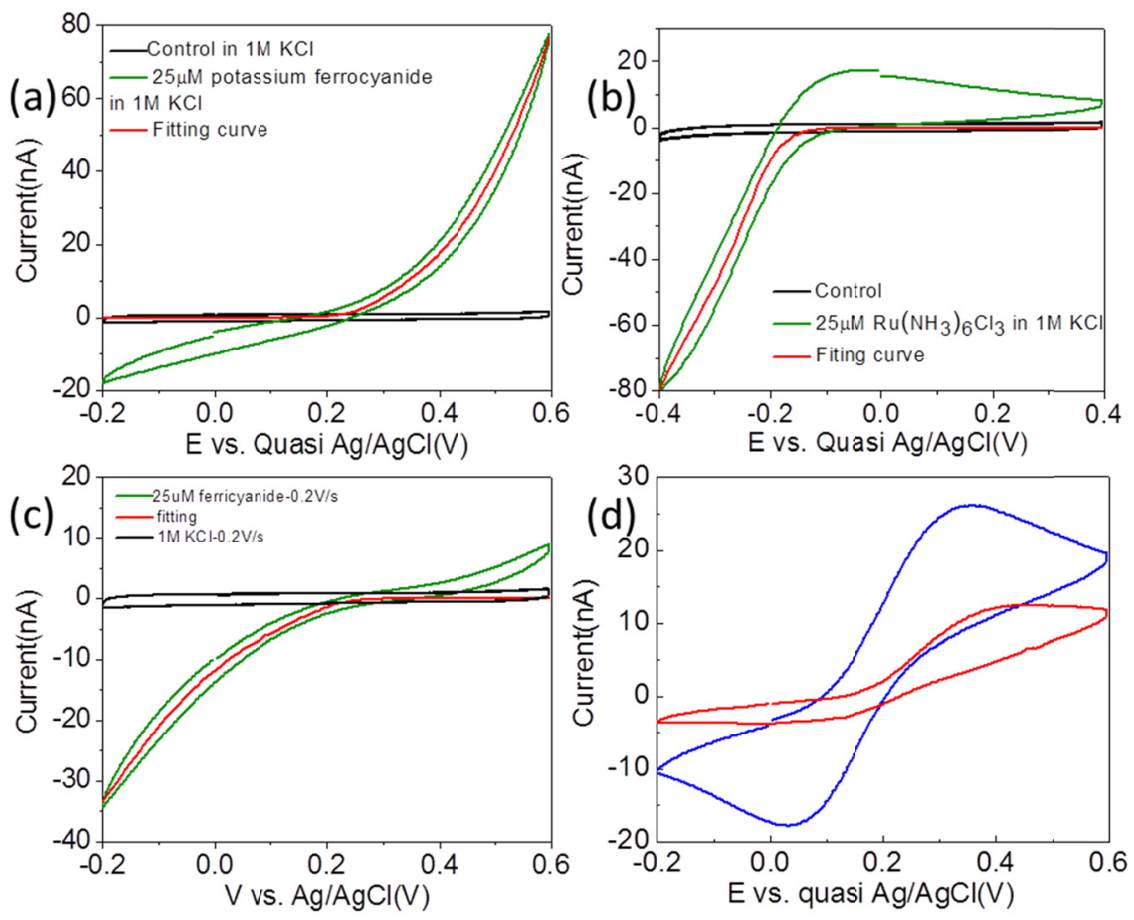

Figure 26: (a) A typical cyclic voltammogram (CV) of $25 \mu \mathrm{M}$ Potassium Ferrocyanide in $1 \mathrm{M}$ KCl. (b) $\mathrm{CV}$ of $25 \mu \mathrm{M} \mathrm{Ru}\left(\mathrm{NH}_{3}\right)_{6} \mathrm{Cl}_{3}$ in $1 \mathrm{M} \mathrm{KCl}$. (c) $\mathrm{CV}$ of $25 \mu \mathrm{M}$ Potassium Ferricyanide in $1 \mathrm{M} \mathrm{KCl}$. For (a), (b) and (c), the red curve is the fitting curve of oxidation current using equation (1). The black curve is a control results in pure $1 \mathrm{M} \mathrm{KCl}$. The potential sweep rate for both $\mathrm{CVs}$ is $200 \mathrm{mV} / \mathrm{s}$. (d) $\mathrm{CV}$ of $20 \mu \mathrm{M} \mathrm{cyc}$ in $1 \mathrm{M} \mathrm{KCl}$ measured before (blue) and after (red) rinsing the CNT thin film surface. The potential scan rate is $25 \mathrm{mV} / \mathrm{s}$. 
Although $\mathrm{Fe}(\mathrm{CN})_{6}^{3-/ 4-}$ ions are widely used as the standard reference system, their electrochemical performance are often affected by the surface states of the CNT surface [32]. Therefore, the redox probe $\mathrm{Ru}\left(\mathrm{NH}_{3}\right)_{6}{ }^{3+}$ was also studied, which is an ideal outersphere redox system and not interacting strongly with the CNT surface. The CV of $25 \mu \mathrm{m}$ $\mathrm{Ru}\left(\mathrm{NH}_{3}\right)_{6} \mathrm{Cl}_{3}$ in $1 \mathrm{M} \mathrm{KCl}$ solution is shown in Figure 26 (b). Reduction current is obvious at negative potential. This shape of $\mathrm{CV}$ is similar to $\mathrm{K}_{3} \mathrm{Fe}(\mathrm{CN})_{6}$ (Figure 26 (c)). A small bump at positive potential is likely caused by the oxidation current of a small number of $\mathrm{Ru}\left(\mathrm{NH}_{3}\right)_{6}{ }^{2+}$ near the CNT surface. The Bulter-Volmer model was also used to fit the reduction current only and a charge transfer rate $0.81 \mathrm{~cm} / \mathrm{s}$ is obtained from the fitting. This rate is high and is comparable with metal electrodes [32].

The interactions between redox molecules and the CNT surface will also affect the shape of the CV. If the molecules transiently stay at the CNT surface, the concentration ratio between reduced and oxidized species near CNT will change. Peak shape CV may be observed in spite of high mass transport rate of molecules near the electrodes surface. We therefore tested redox molecules that could interact strongly with the CNT surface. $\mathrm{Ru}(\mathrm{bpy})_{3} \mathrm{Cl}_{2}$ was first used. On the basis of previous CNT nanopore experiments, the interaction between $\mathrm{Ru}(\mathrm{bpy})_{3}{ }^{2+}$ ion and CNT surface is much stronger than $\mathrm{Fe}(\mathrm{CN})_{6}^{3-/ 4-}[99]$. This is presumably a consequence of the pi-pi stacking interactions between $\mathrm{Ru}(\mathrm{bpy})_{3}{ }^{2+}$ and $\mathrm{CNT}$ surface. Indeed, peak-shape CVs were observed at various potential scan rates, as shown in Figure 27 (a). The peak ratio ipa/ipc is close to 1. This reaction is quasireversible because the peak separation, $\Delta \mathrm{E}_{\mathrm{p}}$, ranges from 100 to $130 \mathrm{mV}$ (Figure 27 (a)). Although $\mathrm{Ru}(\mathrm{bpy})_{3}{ }^{2+}$ has a stronger interaction with CNT surface, the faraday current in the $\mathrm{CV}$ is mainly because of the diffusion of $\mathrm{Ru}(\mathrm{bpy})_{3}{ }^{2+}$ ions. This is 
confirmed by the linear relationship between the cathodic peak current $\left(\mathrm{i}_{\mathrm{pc}}\right)$ or anodic peak current $\left(\mathrm{i}_{\mathrm{pa}}\right)$ and the square root of potential sweep rate (Figure $27(\mathrm{~b})$ ). Therefore, the Nicholson's method [100] was used to calculate $\mathrm{k}^{0}$. The method is applied in the range of $\Delta \mathrm{E}_{\mathrm{p}}$ between $57 \mathrm{mV}$ and $250 \mathrm{mV}$, where the electrode process is progressing from reversible to irreversible. The Nicholson's method describes an empirical correspondence relationship between the peak separation $\left(\Delta \mathrm{E}_{\mathrm{p}}\right)$ and sweep rate $(v)$. The heterogeneous rate constant $\left(\mathrm{k}^{0}\right)$ can be derived from the fitting coefficient of the empirical relationship. The derived value of $\mathrm{k}^{0}$ is $5.4 \times 10^{-2} \mathrm{~cm} / \mathrm{s}$. In addition, the oxidation peak potential of $\mathrm{Ru}(\mathrm{bpy})_{3}{ }^{2+}$ ion is near $0.4 \mathrm{~V}$. This potential is much lower than the observed oxidation peak for $\mathrm{Ru}(\mathrm{bpy})_{3}{ }^{2+}$ at gold or platinum electrodes, where the potential is higher than $1 \mathrm{~V}$. This result demonstrates the electrocatalytic activities of CNT electrode. The catalytical behavior may be the consequence of the stronger interaction between $\mathrm{Ru}(\mathrm{bpy})_{3}{ }^{2+}$ ion and $\mathrm{CNT}$ thin film surface. Besides $\mathrm{Ru}(\mathrm{bpy})_{3}{ }^{2+}$, peak shape $\mathrm{CV}$ curves were also obtained for metalloprotein cytochrome c (cytc) [101] on the aligned SWCNT arrays (see Figure 26 (d)). However, the oxidation peak is much bigger than the reduction peak, which is attributed to the instability of the protein. The $\Delta \mathrm{E}_{\mathrm{p}}$ is also bigger than $200 \mathrm{mV}$. This big peak separation can be attributed to the complex and bigger structure of protein and the slow molecular reorganization upon electron transfer. After rinsing the CNT thin film several times with DI water, the CV was measured again in pure $1 \mathrm{M} \mathrm{KCl}$ solution without cytc. The redox peaks were still visible although there is a 3 fold reduction in peak magnitude. These current are from the adsorbed cytc at CNT surface. In contrast, distinguishable faraday current were observed for $\mathrm{Ru}(\mathrm{bpy})_{3}{ }^{2+}$ and also $\mathrm{Fe}(\mathrm{CN})_{6}^{3-/ 4-}, \mathrm{Ru}\left(\mathrm{NH}_{3}\right)_{6}{ }^{3+/ 2+}$ ions after the same rinsing step. Therefore the adsorption 
of proteins is the strongest among the tested molecules, which is likely a result of the hydrophobic-hydrophobic interactions. This is consistent with the observation by atomic force microscopy (AFM) imaging [102].
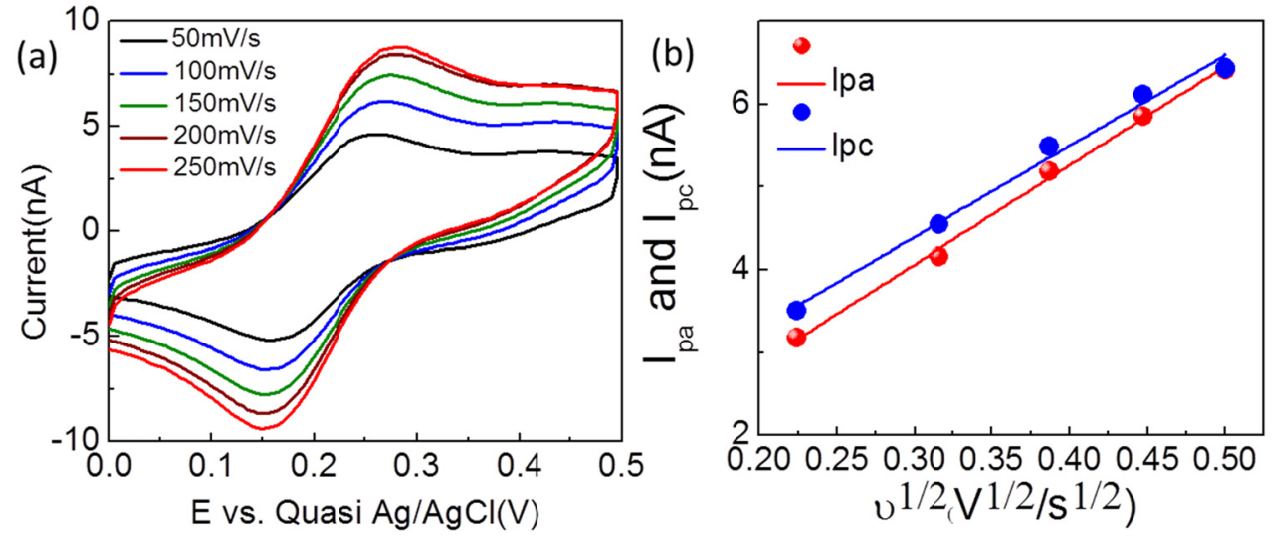

Figure 27: (a) Cyclic voltammograms of $25 \mu \mathrm{M} \mathrm{Ru}(\mathrm{bpy})_{3} \mathrm{Cl}_{2}$ in $1 \mathrm{M} \mathrm{KCl}$ measured at different potential scan rates. (b) The plot of the cathodic peak current $\left(i_{p c}\right)$ and anodic peak current $\left(i_{p a}\right)$ versus the square root of the potential scan rate $\left(v^{1 / 2}\right)$. The solid lines are linear fits to the experimental data.

\subsubsection{Biosensing performance}

To demonstrate the biosensing capability of this device, dopamine was used as the model molecule. Dopamine (DA) is an important neurotransmitter in the central nervous system and is directly responsible for a number of diseases including Parkinson's disease. Therefore, it is important to quantitatively detect DA in human body fluid. DA can be electrochemically oxidized and the oxidation process is irreversible. Figure 28 (a) showed a typical CV curve which was obtained in the experiment with $25 \mu \mathrm{M}$ dopamine in $1 \mathrm{M} \mathrm{KCl}$. Bulter-Volmer model was also used to fit the oxidative current and a standard transfer rate of $5.2 \times 10^{-2} \mathrm{~cm} / \mathrm{s}$ is obtained. 
Subsequently, the EC current was studied at $\mathrm{E}=0.6 \mathrm{~V}$ as a function of dopamine concentration in 1x PBS buffer solution, which is closer to the physiological condition. The results are shown in Figure 28 (b). At low concentration $(\mathrm{c}<20 \mu \mathrm{M})$, the current at $\mathrm{E}=0.6 \mathrm{~V}$ increases proportionally to dopamine concentration. The slope of the linear fitting line is about $0.44 \mathrm{nA} / \mu \mathrm{M}$. The detection limit is about $100 \mathrm{nM}$, where the signal noise ratio is above $3 \sigma$ ( $\sigma$ is the standard deviation of current). At concentration higher than $100 \mu \mathrm{M}$, the slope is obviously reduced and the current becomes less sensitive to dopamine concentration. The saturation should be attributed to the limited CNT thin film surface area. It was found that the CNT thin film with higher area density shows saturation at higher concentration. This suggests a way to enlarge the dynamic range of the sensor by adjusting CNT thin film area density.


Figure 28: (a) A typical cyclic voltammetry (CV) of $25 \mu \mathrm{M}$ dopamine in $1 \mathrm{M} \mathrm{KCl}$. The red curve is the fitting curve using equation (1). The black curve is a control results in pure $1 \mathrm{M} \mathrm{KCl}$. The sweep rate 
is $200 \mathrm{mV} / \mathrm{s}$. (b) Electrochemical (EC) current at $\mathrm{E}=\mathbf{0 . 6 \mathrm { V }}$ as a function of dopamine concentration in 1x PBS buffer. The inset shows the linear relationship between EC current and dopamine concentration at low concentration range. (c) The EC current vs. dopamine concentration with (red sphere) and without (blue sphere) $3 \%(w / w)$ BSA in 1xPBS buffer. The inset shows typical CVs of $1 \mathrm{mM}$ dopamine with (red line) and without (blue line) $3 \%(w / w) ~ B S A$ in 1xPBS buffer. (d) The EC current (at $\mathbf{E}=\mathbf{0 . 6}$ ) versus time when various concentration of dopamine is added into the solution

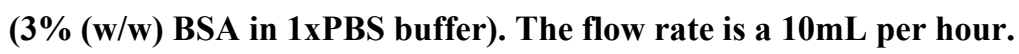

I further investigated the voltammetric behavior of dopamine under conditions that mimic an in vivo environment, i.e., $3 \%(\mathrm{w} / \mathrm{w})$ Bovine serum albumin (BSA) in $1 \mathrm{x}$ PBS solution. Albumin is the most abundant protein in blood plasma and it is often used to evaluate bio-fouling [94]. The CV measurement of dopamine was repeated with the presence of 3\% BSA. A typical result is shown in the inset of Figure 28 (c). The CV shape is the same but the current magnitude is reduced. The EC current at $\mathrm{E}=0.6 \mathrm{~V}$ was compared at different dopamine concentration with and without the presence of $3 \%$ BSA. As shown in Figure 28 (c), a 30\%-40\% current decrease is observed. The reduced current is likely caused by the loss of effective working electrode area owning to non-specific BSA adsorption. It is worth to note that the EC current is quite stable and no reduction in the EC current is observed after ten consecutive potential sweeps even in the presence of BSA.

I also studied the flow sensing capability of the device. To perform this experiment, a syringe pump was used to drive solution continuously flowing over the CNT thin film at a constant flow rate. The current change over time was monitored when various concentrations of dopamine solutions were driven through the CNT thin film surface. As shown in Figure 28 (d), stepwise current increase was observed when the 
concentration of dopamine was increased in the flowing solution with a flow rate $10 \mathrm{ml}$ per hour. The lowest concentration can be reliably detected in flow sensing setup is $0.5 \mu \mathrm{M}$. The response time to the concentration change is in millisecond time range. As the result of low surface coverage, the device $\mathrm{R}_{\mathrm{s}} \mathrm{C}$ (where $\mathrm{C}$ is interfacial capacitance) time constant is small and is in microsecond time range. Therefore, the response time shown here is mainly limited by the mass transport speed, i.e., the flow rate. It is clear from Figure 28 (d) that the EC current did not decrease in a period of 100 seconds. A side view scheme of the flow sensing cell is shown in Figure 29(a). The stability of the EC current was also tested over a much longer time period ( 1000 seconds) when detecting 2,5 and $8 \mu \mathrm{m}$ of dopamine with the presence of BSA. No reduction in current is observed (see Figure 29 (b)). These results demonstrated the nice anti-fouling capability of the CNT thin film. The current versus time plot also demonstrated the quantitatively continuous sensing capability of the device.
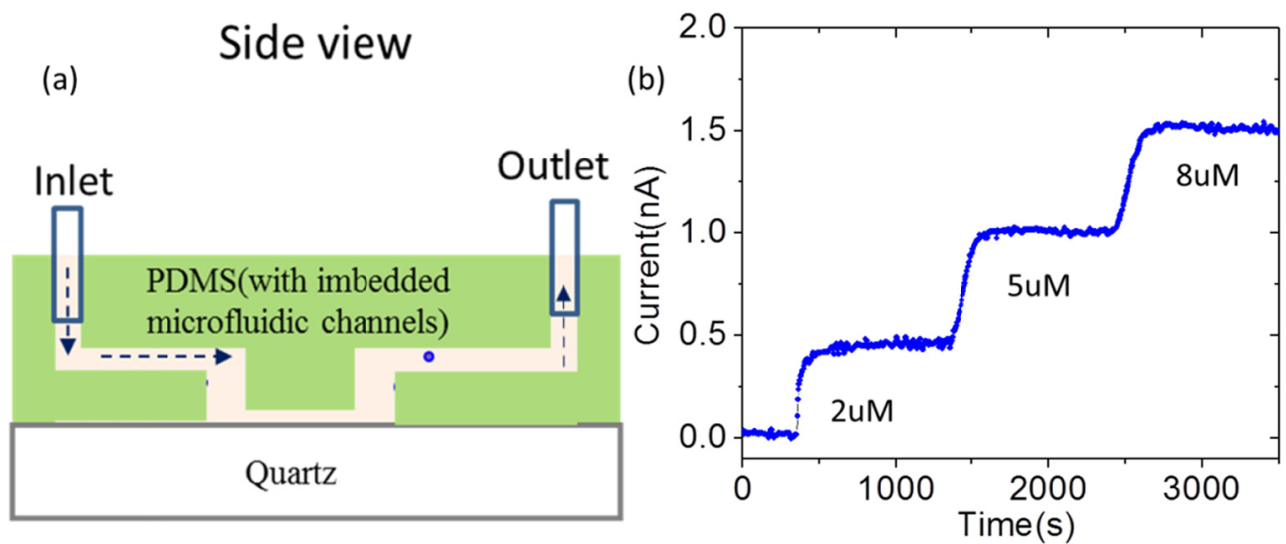

Figure 29: (a) A side-view scheme of the flow sensing cell. (b) The $E C$ current $(E=0.6)$ versus time when 2,5 and $8 \mu \mathrm{M}$ of dopamine is added into the solution (3\% (w/w) BSA in 1xPBS buffer). The flow rate is at $0.5 \mathrm{~mL}$ per hour. 


\subsection{Conclusions}

In summary, an electrochemical sensing device has been successfully fabricated using CVD grown aligned SWCNT arrays on quartz substrate. 2-terminal and 3 terminal electrical measurements have been performed on the sample used in this research, no gating effect was observed. As the result of the extremely low surface coverage and alignment, these CNT arrays show unique electrochemical properties. The electron transfer kinetics of several redox molecules have been studied at the pristine CNT thin film surface. Medium to high charge transfer rates have been observed. The electrochemical performance of redox molecule and protein was also obviously affected by the interaction between molecules and the CNT surface. Dopamine has also been used as a model system. The devices show fast response, high stability and good sensitivity to dopamine even in the presence of $3 \%$ BSA. Here, the CNT thin film was unmodified and untreated. I expect to get enhanced specificity (as well as sensitivity) by modifying different recognition molecules at the CNT surface. The device is almost fully transparent, which can be easily combined with optical manipulation and detection methods. 


\section{CHAPTER 6}

\section{Summary}

Single-walled carbon nanotubes (SWCNTs) have been applied and or been evaluated for use in many fields by employing their mechanical, electrical, optical and electrochemical properties. SWCNTs synthesized by CVD have also emerged into engineering fields such as textile, field emission, chemical/biological sensing, electrical cable/wire manufacturing, energy storage, and solar cell construction. The application of SWCNTs incorporates their properties which related to their sizes, orientation/alignment, and unique electrical structures. The capability of engineering a large variety of useful technological applications will be impacted by controllable SWCNT synthesis at low cost and assembling the nanotube into novel materials with unique and accurately characterized properties including mechanical, electrical, optical, and electrochemical properties. Microelectronics and even medical applications will profit from developing advanced nanotube synthesis process and properties study methods.

I have carried out a successive research on SWCNT in our research group in the past few years including controlled synthesis, mechanical and electrochemical properties studies, and biosensing application of the synthesized sample. The characterized samples acquired from the engineered synthesis process were used to help improve the controllability/accuracy of sample property measurements. The conclusions drawn from the property studies and data analysis were used to evaluate the application potentials of the studied materials.

Specifically, by calibrating the CVD process, horizontally aligned SWCNT arrays have been successfully synthesized on the St-cut quartz substrate with low-defects, 
uniform diameter, and good alignment. The alignment control has been studied and the optimum condition for the alignment was summarized. The structure, morphology, and quality of the synthesized products were characterized using various tools such as scanning electron microscopy (SEM), atomic force microscopies (AFM) and Raman spectroscopy. The advantages of my sample for property studies were indicated.

In the mechanical property study, a simple method and a detailed model have been developed to study the mechanical properties of individual SWCNTs in their radial direction by using tapping mode and contact mode AFM imaging. This method greatly increased the accuracy of radial elasticity measurement of individual SWCNT by avoiding the commonly used nanoindentation method. I have developed an improved theoretical model on the basis of Hertzian theory to analyze the collected data and compared the analytical result with two different theoretical computation results for the first time in this research. The agreement and disagreement with these two theories were elaborated in details.

An electrical/electrochemical properties study has also been conducted systematically, as well as an application research of the horizontally aligned SWCNTs that have been characterized in this research. Using pristine nanotubes grown on transparent quartz substrate as working electrodes, we have fabricated simple and high performance devices and studied the electrochemical activities of redox probes $\mathrm{Fe}(\mathrm{CN})_{6}^{3-}$ ${ }^{14-}, \mathrm{Ru}\left(\mathrm{NH}_{3}\right)_{6}{ }^{3+}, \mathrm{Ru}(\mathrm{bpy})_{3}{ }^{2+}$ and protein cytochrome c. It is found that because the surface coverage of CNTs is extremely low and aligned, the shape of cyclic voltammograms of these molecules in stationary solution is strongly affected by the mass transport rate of molecules and the interactions between molecules and the SWCNT surface. The 
electrochemical flow sensing capability of the device has also been investigated for detecting neurotransmitter dopamine at physiological conditions with the presence of Bovine serum albumin. Good sensitivity, fast response, high stability and anti-fouling capability are observed. Therefore, this device shows great potential for sensing applications in complicated solution.

With newly arisen questions and challenges, research in this field underlines the need for synthesizing SWCNTs with precisely controlled characteristics, designing and conducting precise measurement of sample properties, as well as the property enhancement for applications. My possible future research includes topics such as the scale-up synthesis of SWCNTs with controlled properties, modifying different recognition molecules at the CNT surface to get enhanced specificity (as well as sensitivity) in biosensing, etc. Furthermore, the fully transparent sample of mine can be easily combined with optical manipulation and detection methods. In this endeavor, I will keep accumulating my knowledge in order to further contribute to the SWCNT research in synthesis, property study and applications. 


\section{References}

1. Wang, X.S., et al., Fabrication of Ultralong and Electrically Uniform SingleWalled Carbon Nanotubes on Clean Substrates. Nano Letters, 2009. 9(9): p. 3137-3141.

2. Iijima, S., Helical Microtubules of Graphitic Carbon. Nature, 1991. 354(6348): p. 56-58.

3. Iijima, S. and T. Ichihashi, Single-Shell Carbon Nanotubes of 1-Nm Diameter. Nature, 1993. 363(6430): p. 603-605.

4. Iijima, S. and T. Ichihashi, Single-Shell Carbon Nanotubes of 1-Nm Diameter (Vol 363, Pg 603, 1993). Nature, 1993. 364(6439): p. 737-737.

5. Yu, M.F., et al., Tensile loading of ropes of single wall carbon nanotubes and their mechanical properties. Physical Review Letters, 2000. 84(24): p. 55525555 .

6. Baddour, C. and C. Briens, Carbon nanotube synthesis: A review. International Journal of Chemical Reactor Engineering, 2005. 3.

7. Robertson, J., Growth of nanotubes for electronics. Materials Today, 2007. 10(12): p. 36-43.

8. Palaci, I., et al., Radial elasticity of multiwalled carbon nanotubes. Physical Review Letters, 2005. 94(17).

9. Kratschmer, W., et al., Solid C-60 - a New Form of Carbon. Nature, 1990. 347(6291): p. 354-358.

10. Berber, S., Y.K. Kwon, and D. Tomanek, Unusually high thermal conductivity of carbon nanotubes. Physical Review Letters, 2000. 84(20): p. 4613-4616.

11. Neupane, S., et al., Synthesis and field emission properties of vertically aligned carbon nanotube arrays on copper. Carbon, 2012. 50(7): p. 2641-2650.

12. Foroughi, J., et al., Torsional Carbon Nanotube Artificial Muscles. Science, 2011. 334(6055): p. 494-497.

13. Kroto, H. and H. Terrones, Carbon nanotubes and related structures - new materials for the twenty-first century. Interdisciplinary Science Reviews, 2000. 25(1): p. 78-80.

14. Lu, J.P., Elastic properties of carbon nanotubes and nanoropes. Physical Review Letters, 1997. 79(7): p. 1297-1300. 
15. Dai, H.J., E.W. Wong, and C.M. Lieber, Probing electrical transport in nanomaterials: Conductivity of individual carbon nanotubes. Science, 1996. 272(5261): p. 523-526.

16. de Pablo, P.J., et al., Mechanical and electrical properties of nanosized contacts on single-walled carbon nanotubes. Advanced Materials, 2000. 12(8): p. 573-576.

17. Shen, L.X. and J. Li, Transversely isotropic elastic properties of single-walled carbon nanotubes. Physical Review B, 2004. 69(4).

18. Liew, K.M. and Y.Z. Sun, Elastic properties and pressure-induced structural transitions of single-walled carbon nanotubes. Physical Review B, 2008. 77(20).

19. Tu, Z.C. and Z.C. Ou-Yang, Elastic theory of low-dimensional continua and its applications in bio- and nano-structures (vol 5, pg 422, 2008). Journal of Computational and Theoretical Nanoscience, 2008. 5(6): p. 1192-1192.

20. Minary-Jolandan, M. and M.F. Yu, Reversible radial deformation up to the complete flattening of carbon nanotubes in nanoindentation. Journal of Applied Physics, 2008. 103(7).

21. Shen, W.D., et al., Investigation of the radial compression of carbon nanotubes with a scanning probe microscope. Physical Review Letters, 2000. 84(16): p. 3634-3637.

22. Wang, H.Y., M. Zhao, and S.X. Mao, Radial moduli of individual single-walled carbon nanotubes with and without electric current flow. Applied Physics Letters, 2006. 89(21).

23. Muthaswami, L., et al., Variation of radial elasticity in multiwalled carbon nanotubes. Nano Letters, 2007. 7(12): p. 3891-3894.

24. Yao, Z., C.L. Kane, and C. Dekker, High-field electrical transport in single-wall carbon nanotubes. Physical Review Letters, 2000. 84(13): p. 2941-2944.

25. de Pablo, P.J., et al., Nonlinear resistance versus length in single-walled carbon nanotubes. Physical Review Letters, 2002. 88(3).

26. Andriotis, A.N., M. Menon, and L. Chernozatonskii, Nonlinear resistance dependence on length in single-wall carbon nanotubes. Nano Letters, 2003. 3(2): p. 131-134.

27. de Pablo, P.J., et al., Performing current versus voltage measurements of singlewalled carbon nanotubes using scanning force microscopy. Applied Physics Letters, 2002. 80(8): p. 1462-1464. 
28. Barboza, A.P.M., et al., Deformation induced semiconductor-metal transition in single wall carbon nanotubes probed by electric force microscopy. Physical Review Letters, 2008. 100(25).

29. Cao, J., Q. Wang, and H.J. Dai, Electromechanical properties of metallic, quasimetallic, and semiconducting carbon nanotubes under stretching. Physical Review Letters, 2003.90(15).

30. Park, J.Y., et al., Electron-phonon scattering in metallic single-walled carbon nanotubes. Nano Letters, 2004. 4(3): p. 517-520.

31. Gomez-Navarro, C., J.J. Saenz, and J. Gomez-Herrero, Conductance oscillations in squashed carbon nanotubes. Physical Review Letters, 2006. 96(7).

32. McCreery, R.L., Advanced carbon electrode materials for molecular electrochemistry. Chemical Reviews, 2008. 108(7): p. 2646-2687.

33. Jacobs, C.B., M.J. Peairs, and B.J. Venton, Review: Carbon nanotube based electrochemical sensors for biomolecules. Analytica Chimica Acta, 2010. 662(2): p. 105-127.

34. Heller, I., et al., Individual single-walled carbon nanotubes as nanoelectrodes for electrochemistry. Nano Letters, 2005. 5(1): p. 137-142.

35. Souha, H., et al., Effects of a Silicon-Oxide Layer on Reactivity of Silicon with Copper(I) Chloride. Journal of Materials Science, 1989. 24(5): p. 1767-1771.

36. Daraio, C., et al., Impact response by a foamlike forest of coiled carbon nanotubes. Journal of Applied Physics, 2006. 100(6).

37. Du, F.M., J.E. Fischer, and K.I. Winey, Effect of nanotube alignment on percolation conductivity in carbon nanotube/polymer composites. Physical Review B, 2005. 72(12).

38. Hu, L., D.S. Hecht, and G. Grüner, Percolation in Transparent and Conducting Carbon Nanotube Networks. Nano Letters, 2004. 4(12): p. 2513-2517.

39. Okuda, S., et al., Horizontally Aligned Carbon Nanotubes on a Quartz Substrate for Chemical and Biological Sensing. Journal of Physical Chemistry C, 2012. 116(36): p. 19490-19495.

40. Bethune, D.S., et al., Cobalt-Catalyzed Growth of Carbon Nanotubes with SingleAtomic-Layerwalls. Nature, 1993. 363(6430): p. 605-607.

41. Guo, T., et al., Self-Assembly of Tubular Fullerenes. Journal of Physical Chemistry, 1995. 99(27): p. 10694-10697. 
42. Guo, T., et al., Catalytic Growth of Single-Walled Nanotubes by Laser Vaporization. Chemical Physics Letters, 1995. 243(1-2): p. 49-54.

43. Nikolaev, P., et al., Gas-phase catalytic growth of single-walled carbon nanotubes from carbon monoxide. Chemical Physics Letters, 1999. 313(1-2): p. 91-97.

44. Tang, Z.K., et al., Superconductivity in 4 angstrom single-walled carbon nanotubes. Science, 2001. 292(5526): p. 2462-2465.

45. Jachlewski, T. and R.J. Diefendorf, Chemical Vapor-Deposition of Carbon Matrices. Carbon, 1982. 20(2): p. 149-149.

46. Cummings, D.F. and R.J. Diefendorf, The Chemical Vapor-Deposition of Carbon on Graphite Surfaces. Carbon, 1984. 22(2): p. 214-215.

47. Joseyacaman, M., et al., Catalytic Growth of Carbon Microtubules with Fullerene Structure. Applied Physics Letters, 1993. 62(2): p. 202-204.

48. Maruyama, S., et al., Low-temperature synthesis of high-purity single-walled carbon nanotubes from alcohol. Chemical Physics Letters, 2002. 360(3-4): p. 229-234.

49. Melechko, A.V., et al., Vertically aligned carbon nanofibers and related structures: Controlled synthesis and directed assembly. Journal of Applied Physics, 2005. 97(4).

50. Terrones, M., Science and technology of the twenty-first century: Synthesis, properties and applications of carbon nanotubes. Annual Review of Materials Research, 2003. 33: p. 419-501.

51. Journet, C., et al., Large-scale production of single-walled carbon nanotubes by the electric-arc technique. Nature, 1997. 388(6644): p. 756-758.

52. Dittmer, S., J. Svensson, and E.E.B. Campbell, Electric field aligned growth of single-walled carbon nanotubes. Current Applied Physics, 2004. 4(6): p. 595-598.

53. Krupke, R., et al., Thin films of metallic carbon nanotubes prepared by dielectrophoresis. Advanced Materials, 2006. 18(11): p. 1468-+.

54. Li, X.L., et al., Langmuir-Blodgett assembly of densely aligned single-walled carbon nanotubes from bulk materials. Journal of the American Chemical Society, 2007. 129(16): p. 4890-+.

55. Cao, Q. and J.A. Rogers, Ultrathin Films of Single-Walled Carbon Nanotubes for Electronics and Sensors: A Review of Fundamental and Applied Aspects.

Advanced Materials, 2009. 21(1): p. 29-53. 
56. Liu, J., et al., Chirality-controlled synthesis of single-wall carbon nanotubes using vapour-phase epitaxy. Nature Communications, 2012. 3.

57. Kocabas, C., et al., Guided growth of large-scale, horizontally aligned arrays of single-walled carbon nanotubes and their use in thin-film transistors. Small, 2005. 1(11): p. 1110-1116.

58. Rutherglen, C., D. Jain, and P. Burke, Nanotube electronics for radiofrequency applications. Nature Nanotechnology, 2009. 4(12): p. 811-819.

59. Kang, S.J., et al., High-performance electronics using dense, perfectly aligned arrays of single-walled carbon nanotubes. Nature Nanotechnology, 2007. 2(4): p. 230-236.

60. McNicholas, T.P., et al., Density Enhancement of Aligned Single-Walled Carbon Nanotube Thin Films on Quartz Substrates by Sulfur-Assisted Synthesis. Nano Letters, 2009. 9(10): p. 3646-3650.

61. Ding, L., et al., Selective Growth of Well-Aligned Semiconducting Single-Walled Carbon Nanotubes. Nano Letters, 2009. 9(2): p. 800-805.

62. Zhang, Y.G., et al., Electric-field-directed growth of aligned single-walled carbon nanotubes. Applied Physics Letters, 2001. 79(19): p. 3155-3157.

63. Ural, A., Y.M. Li, and H.J. Dai, Electric-field-aligned growth of single-walled carbon nanotubes on surfaces. Applied Physics Letters, 2002. 81(18): p. 34643466.

64. Chiu, C.C., et al., Tip-to-tip growth of aligned single-walled carbon nanotubes under an electric field. Journal of Crystal Growth, 2006. 290(1): p. 171-175.

65. Huang, S.M., et al., Growth mechanism of oriented long single walled carbon nanotubes using "fast-heating" chemical vapor deposition process. Nano Letters, 2004. 4(6): p. 1025-1028.

66. Zheng, L.X., et al., Ultralong single-wall carbon nanotubes. Nature Materials, 2004. 3(10): p. 673-676.

67. Jin, Z., et al., Ultralow feeding gas flow guiding growth of large-scale horizontally aligned single-walled carbon nanotube arrays. Nano Letters, 2007. 7(7): p. 2073-2079.

68. Rutkowska, A., et al., Horizontal Alignment of Chemical Vapor-Deposited SWNTs on Single-Crystal Quartz Surfaces: Further Evidence for Epitaxial Alignment. Journal of Physical Chemistry C, 2009. 113(39): p. 17087-17096. 
69. Geblinger, N., A. Ismach, and E. Joselevich, Self-organized nanotube serpentines. Nature Nanotechnology, 2008. 3(4): p. 195-200.

70. Huang, L.M., et al., Cobalt ultrathin film catalyzed ethanol chemical vapor deposition of single-walled carbon nanotubes. Journal of Physical Chemistry B, 2006. 110(23): p. 11103-11109.

71. Xiao, J.L., et al., Alignment Controlled Growth of Single-Walled Carbon Nanotubes on Quartz Substrates. Nano Letters, 2009. 9(12): p. 4311-4319.

72. Yang, Y.H. and W.Z. Li, Radial elasticity of single-walled carbon nanotube measured by atomic force microscopy. Applied Physics Letters, 2011. 98(4).

73. Kocabas, C., et al., Improved synthesis of aligned arrays of single-walled carbon nanotubes and their implementation in thin film type transistors. Journal of Physical Chemistry C, 2007. 111(48): p. 17879-17886.

74. Kocabas, C., M. Shim, and J.A. Rogers, Spatially selective guided growth of highcoverage arrays and random networks of single-walled carbon nanotubes and their integration into electronic devices. Journal of the American Chemical Society, 2006. 128(14): p. 4540-4541.

75. Ibrahim, I., et al., Optimizing substrate surface and catalyst conditions for high yield chemical vapor deposition grown epitaxially aligned single-walled carbon nanotubes. Carbon, 2011. 49(15): p. 5029-5037.

76. Heffelfinger, J.R., M.W. Bench, and C.B. Carter, Steps and the structure of the (0001)alpha-alumina surface. Surface Science, 1997. 370(1): p. L168-L172.

77. Jeon, S., et al., Growth of Serpentine Carbon Nanotubes on Quartz Substrates and Their Electrical Properties. Nano Research, 2008. 1(5): p. 427-433.

78. Feng, C.Q., et al., Nanobarrier-Terminated Growth of Single-Walled Carbon Nanotubes on Quartz Surfaces. Nano Research, 2009. 2(10): p. 768-773.

79. Dresselhaus, M.S., et al., Raman spectroscopy of carbon nanotubes. Physics Reports-Review Section of Physics Letters, 2005. 409(2): p. 47-99.

80. Wu, C.C., et al., Efficient organic blue-light-emitting devices with double confinement on terfluorenes with ambipolar carrier transport properties. Advanced Materials, 2004. 16(1): p. 61-+.

81. Ajayan, P.M. and J.M. Tour, Materials science - Nanotube composites. Nature, 2007. 447(7148): p. 1066-1068.

82. Cao, Q., et al., Medium-scale carbon nanotube thin-film integrated circuits on flexible plastic substrates. Nature, 2008. 454(7203): p. 495-U4. 
83. Close, G.F., et al., A 1 GHz integrated circuit with carbon nanotube interconnects and silicon transistors. Nano Letters, 2008. 8(2): p. 706-709.

84. Barboza, A.P.M., H. Chacham, and B.R.A. Neves, Universal Response of SingleWall Carbon Nanotubes to Radial Compression. Physical Review Letters, 2009. 102(2).

85. DeBorde, T., et al., Identifying Individual Single-Walled and Double-Walled Carbon Nanotubes by Atomic Force Microscopy. Nano Letters, 2008. 8(11): p. 3568-3571.

86. Jiang, J.W., J.S. Wang, and B.W. Li, Young's modulus of graphene: A molecular dynamics study. Physical Review B, 2009. 80(11).

87. Chen, W.H., H.C. Cheng, and Y.L. Liu, Radial mechanical properties of singlewalled carbon nanotubes using modified molecular structure mechanics. Computational Materials Science, 2010. 47(4): p. 985-993.

88. Sun, D.Y., et al., Pressure-induced hard-to-soft transition of a single carbon nanotube. Physical Review B, 2004. 70(16).

89. Heller, I., et al., Individual Single-Walled Carbon Nanotubes as Nanoelectrodes for Electrochemistry. Nano Letters, 2004. 5(1): p. 137-142.

90. Wang, J., Carbon-Nanotube Based Electrochemical Biosensors: A Review. Electroanalysis, 2005. 17(1): p. 7-14.

91. Hu, L., D.S. Hecht, and G. Grüner, Carbon Nanotube Thin Films: Fabrication, Properties, and Applications. Chemical Reviews, $2010.110(10)$ : p. 5790-5844.

92. Wu, Z., et al., Transparent, Conductive Carbon Nanotube Films. Science, 2004. 305(5688): p. 1273-1276.

93. Bertoncello, P., et al., Trace Level Cyclic Voltammetry Facilitated by SingleWalled Carbon Nanotube Network Electrodes. Journal of the American Chemical Society, 2007. 129(36): p. 10982-10983.

94. Dumitrescu, I., et al., Ultrathin Carbon Nanotube Mat Electrodes for Enhanced Amperometric Detection. Advanced Materials, 2009. 21(30): p. 3105-3109.

95. Du, F., J.E. Fischer, and K.I. Winey, Effect of nanotube alignment on percolation conductivity in carbon nanotube/polymer composites. Physical Review B, 2005. 72(12): p. 121404.

96. Okuda, S., et al., Horizontally Aligned Carbon Nanotubes on a Quartz Substrate for Chemical and Biological Sensing. The Journal of Physical Chemistry C, 2012. 116(36): p. 19490-19495. 
97. Yaglioglu, O., et al., Method of characterizing electrical contact properties of carbon nanotube coated surfaces. Review of Scientific Instruments, 2006. 77(9): p. 095105.

98. Güell, A.G., et al., Quantitative nanoscale visualization of heterogeneous electron transfer rates in $2 D$ carbon nanotube networks. Proceedings of the National Academy of Sciences, 2012. 109(29): p. 11487-11492.

99. Krishnakumar, P., et al., Mass transport through vertically aligned large diameter MWCNTs embedded in parylene. Nanotechnology, 2012. 23(45): p. 455101.

100. Allen J. Bard and L.R. Faulkner, Electrochemical Methods: Fundamentals and Applications. 2000: Wiley, John \& Sons, Incorporated.

101. Salamon, Z., J.T. Hazzard, and G. Tollin, Direct measurement of cyclic currentvoltage responses of integral membrane proteins at a self-assembled lipidbilayer-modified electrode: cytochrome f and cytochrome c oxidase. Proceedings of the National Academy of Sciences, 1993. 90(14): p. 6420-6423.

102. Boussaad, S., et al., In situ detection of cytochrome c adsorption with single walled carbon nanotube device. Chemical Communications, 2003(13): p. 15021503. 
VITA

YUEHAI YANG

\section{EDUCATION}

Florida International University

Miami, FL

Ph. D. in Experimental Condensed Matter Physics

Ocean University of China

M. S. in Computational Condensed Matter Physics

2007-2013

B. S. in Physics (Concentration: Acoustics and Optics)

Qingdao, China

2004-2007

2000-2004

\section{PUBLICATIONS AND PRESENTATIONS}

\section{Peer Review Articles}

-Yuehai Yang, Xuewen Wang, Wenzhi, Li and Jin He "Horizontally Aligned SWCNT Arrays on Quartz for Electrochemical Biosensing"-Manuscript submitted

-Jianyuan Dai, Janak Paudyal, Bhargav Guntupalli, Pingping Liang, Felipe Pubillones, Yuehai Yang, Purushottam Tiwari, Wenzhi Li, Jin He, Xiaotang Wang, and Yi Xiao "Paper-Based Single-Walled Carbon Nanotube Thin Film for Catalytic Detection of Nicotinamide Adenine Dinucleotide in Human Blood"Manuscript submitted

-Suman Neupane, Yuehai Yang, Wenzhi Li, Qingmei Su and Gaohui Du "A Facile Approach for Large-scale Fabrication of Free-standing Vertically Aligned SnO2/CNT Arrays on Conducting Substrate"- Manuscript accepted

-Yuehai Yang and Wenzhi Li "Radial Elasticity of Single-walled Carbon Nanotube Measured by Atomic Force Microscopy” Applied Physics Letters 98, 041901 (2011).

-Garima Thakur, Miodrag Micic, Yuehai Yang, Wenzhi Li, Dania Movia, Silvia Giordani et al. "Conjugated Quantum Dots Inhibit the Amyloid B (1-42) Fibrillation Process" International Journal of Alzheimer's Disease (2011) doi:10.4061/2011/502386.

-Yuehai Yang, Shunle Dong, and Lin Wang "Lattice Dynamics Study of Low Energy Guest-host Coupling in Clathrate Hydrate” Chinese Phys. B 17, 270 (2008)

\section{Book Entry}

-Yuehai Yang (also the corresponding author) and Wenzhi Li "Gas Phase Nanoparticle Formation"_Encyclopedia of Nanotechnology (2012) 10.1007/978-90481-9751-4_358

American Physical Society 2012 March Meeting Boston, MA

- Oral presentation: "Radial Elasticity Measurement of Single-walled Carbon Nanotubes by Atomic Force Microscopy" 